\title{
GEOMETRIZING INFINITE DIMENSIONAL LOCALLY COMPACT GROUPS
}

\author{
CONRAD PLAUT
}

\begin{abstract}
We study groups having invariant metrics of curvature bounded below in the sense of Alexandrov. Such groups are a generalization of Lie groups with invariant Riemannian metrics, but form a much larger class. We prove that every locally compact, arcwise connected, first countable group has such a metric. These groups may not be (even infinite dimensional) manifolds. We show a number of relationships between the algebraic and geometric structures of groups equipped with such metrics. Many results do not require local compactness.
\end{abstract}

\section{Introduction And Main Results}

The traditional notion of infinite dimensional manifold excludes, among other things, all locally compact spaces. This exclusion means that Hilbert's Fifth Problem (Sophus Lie's theory of transformation groups without differentiability assumptions) must, for infinite dimensional groups, be interpreted differently from the way it was for finite dimensional groups. Instead of finding suitable differentiable coordinates for a group, we have to take the problem in a more general sense, for example by seeking invariant geometries associated with groups, which can be used to understand their algebraic structure. In this paper we approach the problem by extending the notion of invariant Riemannian metric on a Lie group to a reasonable class of infinite dimesional groups. Roughly speaking, the "weakly convex" metric we define has curvature bounded below and local convexity on finite dimensional "slices." We believe that groups with invariant weakly convex metrics are a suitable generalization of Lie groups with invariant Riemannian metrics for several reasons. First, every first countable, arcwise connected, locally compact group has such a metric (Theorem 1.1). Second, there is some useful interplay between the geometric and algebraic structures on such groups. In the process of proving Theorem 1.1 we use purely metric arguments to obtain a number of results in this direction, usually assuming neither local compactness nor weak convexity. For example, we show that for bi-invariant metrics, any lower curvature bound implies non-negative curvature, geodesic completeness always holds, and positive cut radius implies that geodesics and 1-parameter subgroups coincide (Section 4). We also obtain various results extending the orthogonal splitting of the tangent space induced from the

Received by the editors February 16, 1994.

1991 Mathematics Subject Classification. Primary 53C70, 22D05; Secondary 22E65.

Key words and phrases. Locally compact groups, Alexandrov curvature, invariant metric.

The author gratefully acknowledges the support of NSF grant DMS-9401302. 
quotient of a Lie group by a closed subgroup (Section 2). We believe the potential for such results is far from exhausted.

A third reason we believe our metrics are appropriate is that a finite dimensional group equipped with a weakly convex metric is isometric to a Lie group with invariant Riemannian metric. This follows from either [4] or [19], [20]. It is also true that a weakly convex metric on any finite dimensional topological space forces it to be a smooth manifold (see below).

All spaces in this paper are Hausdorff and connected; unless otherwise stated, metric spaces are assumed complete, and rectifiable curves are parameterized proportional to arclength. "Dimension" refers to covering dimension. For first countable groups, local connectedness, local arcwise connectedness, and arcwise connectedness are equivalent (cf. [22]).

In the next four paragraphs we will state some basic definitions, classical results, and some recent results about metric spaces of curvature bounded below, an area initiated by A. D. Alexandrov in the 1940's. Good references for the basics of Alexandrov's theory are [24], [2], [17], [7]; basic theory for spaces which are not locally compact, including an extension of the Hopf-Rinow Theorem, may be found in [21]; good references for metric Riemannian geometry are [6] and [11].

A metric space $(X, d)$ is called an inner metric space (also length or intrinsic metric space) if for any two points $x, y \in X, d(x, y)$ is the infimum of the lengths of curves joining $x$ and $y$. The length $L(c)$ of a curve $c$ is measured in the usual way, by summing distances over finer and finer partitions of $c$. In locally compact spaces, a theorem of Cohn-Vossen ([8]) states that there is always a minimal curve joining each pair of points, whose length realizes the distance between them. A geodesic $\gamma$ is a locally minimizing curve; i.e. every point on $\gamma$ lies in the interior of a segment $\sigma$ of $\gamma$ which is minimal between its endpoints. For any number $k$, an inner metric space $X$ has curvature $\geq k$ if every quadruple in $X$ isometrically embeds in $S_{l}^{2}$ for some $l \geq k$. Here $S_{l}^{2}$ denotes the simply connected, 2 dimensional space form of curvature $l$ : a sphere for $l>0$, the plane for $l=0$, and a hyperbolic space for $l<0$. This definition is due to Wald and Berestovskii ([3]), and is equivalent to Alexandrov's in the locally compact case. Curvature bounded below is a generalization of the notion of sectional curvature bounded below in a Riemannian manifold $M$ (cf. [6]). Here we consider $M$ as a metric space with the metric induced by measuring lengths of curves using the Riemannian metric. Piecewise differentiable curves may be equivalently measured using either the Riemannian metric or this induced metric (distance); therefore the induced metric is inner. The Riemannian and metric notions of geodesic are also equivalent in this setting.

For the rest of this discussion we will suppose that $X$ is an inner metric space of curvature bounded below. The angle $\alpha\left(\gamma_{1}, \gamma_{2}\right)$ between any two geodesics $\gamma_{1}, \gamma_{2}$ starting at a common point $p$ in $X$ is measured using the Euclidean cosine law: if $\alpha_{t}=\cos ^{-1}\left(1-\frac{d\left(\gamma_{1}(t), \gamma_{2}(t)\right)^{2}}{2 t^{2}}\right)$ then the curvature bound implies $\lim _{t \rightarrow 0} \alpha_{t}$ exists, and we define this limit to be $\alpha\left(\gamma_{1}, \gamma_{2}\right)$. An important property of angles in $X$ is the usual Euclidean property that the sum of complementary angles is $\pi$.

We will use Alexandrov's triangle comparisons in this paper: any triangle $T$ in $X$ whose sides are formed by minimal curves has the property that the three angles of $T$ are not smaller than the corresponding angles in a triangle $T^{\prime}$ of the same side lengths in $S_{k}^{2}$. Roughly speaking, triangles are always "fatter" in $X$ than in $S_{k}^{2}$; equivalently, geodesics starting at a common point diverge more slowly in $X$ than 
in $S_{k}^{2}$. An immediate consequence of these comparisons is that two geodesics which coincide on an open interval are identical (up to reparameterization) wherever both are defined. Given any point $p \in X$, a geodesic direction at $p$ is an equivalence class of geodesics, where two geodesics are equivalent if they coincide on an open interval. Equivalently, a geodesic direction is a unit (arclength parameterized) geodesic of maximal domain of definition. $X$ is geodesically complete if the maximal domain of definition of every geodesic is $\mathrm{R}$. For any $p \in X, S_{p}$ denotes the space of geodesic directions, which is given the angle metric; the space of directions, $\bar{S}_{p}$, is the metric completion of $S_{p}$. The tangent cone and completed tangent cones, which are the metric cones on $S_{p}$ and $\bar{S}_{p}$, respectively, are denoted by $T_{p}$ and $\bar{T}_{p}$ (cf. [17]). If $X$ is geodesically complete, $\bar{T}_{p}$ is a Hilbert space $([17])$, the cone on its unit sphere $\bar{S}_{p}$. Recall that the Hopf-Rinow Theorem states that a complete Riemannian manifold is always geodesically complete, and every direction is a geodesic direction; in this case $T_{p}=\bar{T}_{p}$ can be identified with the usual tangent space of the underlying smooth manifold; the angle (and distance) are also induced by the Riemannian metric.

The exponential map $\exp _{p}: T_{p} \rightarrow X$ carries radial lines from the cone point (0) to the corresponding geodesics in $X$. In a Riemannian manifold this notion of $\exp _{p}$ coincides with the usual one. Recall that for a Lie group with invariant Riemannian metric, there is also an algebraic exponential map which carries radial lines onto 1-parameter subgroups. For infinite dimensional groups (cf. [14]) the difference between these maps is much greater (even their domains are different); this situation is investigated further in a paper presently in preparation by the author, V.N. Berestovskii, and C. Stallmann.

The cut radius map (cf. [17]) $c_{p}: \bar{S}_{p} \rightarrow \mathrm{R}$ assigns to each direction the maximal length for which the corresponding geodesic (if there is one) is minimal; if there is no corresponding geodesic, the cut radius is 0 . Note that $S_{p}$ is by definition the subset of $\bar{S}_{p}$ on which $c_{p}$ is positive. The cut radius map is semi-continuous but (if the space is not Riemannian) not necessarily continuous (cf. [17]). Two Euclidean disks glued together along their boundaries is a simple example ([1]) of a geodesically complete space of curvature $\geq 0$ which is not Riemannian.

Any Banach space has an induced inner metric, but the metric generally does not have curvature bounded below (unless the norm comes from an inner product, in which case it is immediate from the definition that the curvature is non-negative). In general, curvature bounded below is a very strong condition, which often has powerful geometric and topological consequences.

Definition 1.1. A real valued (possibly not continuous) function $f$ on a subset $Y$ of a topological vector space $\mathcal{V}$ is called weakly positive if for every finite dimensional linear subspace $\mathcal{W}$ of $\mathcal{V},\left.f\right|_{Y \cap \mathcal{W}}$ has a positive lower bound.

Definition 1.2. A metric on a space $X$ is said to be weakly convex if it is a geodesically complete inner metric of curvature $\geq k$ (for some $k$ ) and for each $p \in X$, there is a vector subspace $W_{p}$ of $T_{p}$ such that

1) $\left.c_{p}\right|_{S_{p} \cap W_{p}}$ is weakly positive, and

2) for every $\gamma \in \bar{S}_{p}$ there exist $\gamma_{i} \in W_{p} \cap S_{p}$ such that $\alpha\left(\gamma_{i}, \gamma\right) \rightarrow 0$ and $c_{p}\left(\gamma_{i}\right) \rightarrow c_{p}(\gamma)$.

Condition 2) is a kind of strong density of $W_{p}$, and implies that $W_{p}$ is dense in $\bar{T}_{p}$. This condition also implies that what should be the "normal coordinates" for 
this metric have dense image in $X$. If $X$ is finite dimensional then we immediately have $W_{p}=\bar{T}_{p}$, and $c_{p}$ is positive for all $p$. This implies that $X$ is a smooth manifold ([18]). If $X$ is infinite dimensional and locally compact, we conjecture that $W_{p}$ is always a (noncomplete) proper subspace of $\bar{T}_{p}$, since $c_{p}$ must be positive on a locally convex subset of $W_{p}$ which is not open. However, $W_{p}$ is geometrically as "complete" as $\bar{T}_{p}$ in the sense that the set of points joined to $p$ by a unique geodesic in $W_{p}$ is dense in $X$.

Let $G$ be a topological group. A metric on $G$ is called invariant if it is invariant under left translation, and bi-invariant if it is also invariant under right translation.

Theorem 1.1. Every arcwise connected, first countable, locally compact group $G$ admits an invariant, weakly convex inner metric $d$ of curvature $\geq k$. In addition,

1. Every direction in $\bar{S}_{e}$ is tangent to a unique unit 1-parameter subgroup, and every non-trivial rectifiable 1-parameter subgroup is tangent to a direction.

2. If $d$ is bi-invariant, then $k=0$, every geodesic through $e$ is a 1-parameter subgroup, and every 1-parameter subgroup tangent to a geodesic direction is a geodesic.

3. If $G$ is compact we can take $d$ to be bi-invariant.

Note that arcwise connectedness and first countability are necessary conditions for the existence of any inner metric on $G$. Infinite dimensional locally compact spaces with weakly invariant metric cannot have positive cut radius, since then they would be locally homeomorphic (via $\exp _{e}$ ) to an infinite dimensional Hilbert space (and these are never locally compact). In particular, these spaces cannot have an upper curvature bound in the usual sense.

For the meaning of "tangent" see Definition 2.3. There are non-rectifiable 1parameter subgroups in every such metric on an infinite dimensional locally compact group (see Corollary 5.3 and subsequent comment). Note that even in the compact case, rectifiable 1-parameter subgroups need not be geodesics, because in infinite dimensions the cut radius is not positive, so there are always directions which are not geodesic directions.

Since we will only be treating spaces having a lower curvature bound, we will often refer to a metric of the type described in Theorem 1.1 (invariant or otherwise) as simply a "weakly convex" metric. We denote the identity element of a group $G$ by $e$. When $G$ has an invariant metric, we will often use implicitly the fact that $G$ is homogeneous; i.e. has a transitive group of isometries, namely the left translations $L_{a}(x)=a x$. This allows us to prove local metric conditions only near $e$. When we specify a geodesic $\gamma$ through $e$, it will be understood that $\gamma$ is unit and $\gamma(0)=e$.

A number of the properties of the metric given by Theorem 1.1 are true for more general metrics. In the next theorems we collect some of these results, which are proved in this paper:

Theorem 1.2. Let $G$ be a group with bi-invariant inner metric of curvature $\geq k$. Then

1. G has curvature $\geq 0$,

2. every geodesic through $e$ is a 1-parameter subgroup, and

3. $G$ is geodesically complete.

Theorem 1.3. If $G$ is a group with left invariant inner metric of curvature $\geq k$, then every non-trivial rectifiable 1-parameter subgroup of $G$ is tangent to a direction in $\bar{S}_{e}$. 
In fact a stronger statement than Theorem 1.3, is true, see Corollary 5.1.

We conclude this section with a simple motivating example, which, although very special, is really not too different from what one finds in general. We only sketch our construction here; details carried out in greater generality may be found later in the paper.

Example 1.1. Let $T^{\infty}=S^{1} \times S^{1} \times \cdots$ with the product topology. Then $T^{\infty}$ is a compact, abelian group. $T^{\infty}$ is not a manifold; arbitrarily small neighborhoods are homeomorphic to products of $T^{\infty}$ itself with Euclidean open sets of arbitrarily large dimension. We metrize $T^{\infty}$ by taking the usual metric on each copy of $S^{1}$ but letting the diameter of the $i^{t h}$ copy be $2^{-i}$. We then take the product metric with more and more factors. Each finite product is naturally embedded in its successor; passing to the limit we obtain a bi-invariant inner metric on $T^{\infty}$. Although it is not a manifold, $T^{\infty}$, metrized this way, "ought" to be a flat Riemannian manifold. Any triangle of strictly minimal curves (uniquely joining their endpoints) embeds isometrically in the plane, i.e. the "sectional curvature" is 0. However, there is no neighborhood in which all minimal curves are unique between their endpoints. Thus, although there is a lower curvature bound of 0 in Alexandrov's sense, there is no upper curvature bound. On the other hand, the lower curvature bound gives us a "tangent space" which is a separable Hilbert space, and an exponential map defined on this Hilbert space. From the standpoint of Riemannian geometry, this exponential map is about as reasonable as one could expect in this generalitynamely, it is injective (and surjective onto a dense subset) when restricted to a relatively compact, locally convex (but non-open) subset of a non-complete infinite dimensional inner product space. This exponential map differs from the algebraic one ([14]) and in particular does not "cover" non-rectifiable 1-parameter subgroups.

\section{Quotients}

Definition 2.1. Let $(X, d)$ be a complete metric space and $G$ be a group of isometries of $X$ whose orbits are all closed. The orbit space $X / G$ is given the induced met$\operatorname{ric} d\left(G x_{1}, G x_{2}\right)=\inf \left\{d\left(y_{1}, y_{2}\right): y_{1} \in G x_{1}, y_{2} \in G x_{2}\right\}=\inf \left\{d\left(x_{1}, y_{2}\right): y_{2} \in G x_{2}\right\}$. The projection map $p: X \rightarrow X / G$ is defined by $p(x)=G x$.

In this paragraph we recall some of the basic results of Berestovskii ([4]) on orbit spaces of inner metric spaces. If $X$ is an inner metric space then $X / G$ is also an inner metric space. Roughly speaking, an "almost" midpoint for orbits $G x_{1}$ and $G x_{2}$ is the orbit of a point $x_{3}$ which is almost a midpoint between points almost realizing the distance between $G x_{1}$ and $G x_{2}$. Such "almost" midpoints can be used to construct "almost" minimal curves between $G x_{1}$ and $G x_{2}$ (cf. also [24],[10], [17]). If $x_{3}$ is a midpoint between $x_{1}$ and $x_{2}$ then $G x_{3}$ is a midpoint between $G x_{1}$ and $G x_{2}$.

Definition 2.2. A metric space $X$ is finitely compact if closed bounded subsets of $X$ are compact.

If $X$ is an inner metric space, finite compactness and local compactness is equivalent ([8]). If the orbits of $G$ are finitely compact in the induced metric, then the distance between orbits is always realized as the distance between two points. In this case, a curve $\gamma$ is minimal from $G x_{1}$ to $G x_{2}$ if and only if $\gamma=p(\Gamma)$, where $\Gamma$ is minimal from $y_{1} \in G x_{1}$ to $y_{2} \in G x_{2}$ such that $d\left(y_{1}, y_{2}\right)=d\left(G x_{1}, G x_{2}\right)$. We will call $\Gamma$ a lift of $\gamma ;\left.p\right|_{\Gamma}$ is an isometry. By translating with an isometry we can assume 
the lift starts at any point in $G x_{1}$. If $X$ has curvature $\geq k$ then it is immediate from the curvature definition that $X / G$ also has curvature $\geq k$. In this case, given minimal curves $\gamma_{1}, \gamma_{2}$ in $X / G$ starting at a single point and lifts $\Gamma_{1}, \Gamma_{2}$ starting at a single point, it is immediate from the definition of the angle and the fact that $p$ is distance decreasing, that $\alpha\left(\gamma_{1}, \gamma_{2}\right) \leq \alpha\left(\Gamma_{1}, \Gamma_{2}\right)$. We note here that it is easy to verify that the quotient metric coincides with the induced Riemannian metric in case $p$ is a Riemannian submersion ([16]). We say that a geodesic is extendable past a point if it can be extended as a geodesic beyond the point.

From now on we will assume that $X$ is an inner metric space and $G$ is a group of isometries of $X$ with finitely compact orbits. The latter assumption is automatic if $X$ is locally compact and the orbits of $G$ are closed.

Lemma 2.1. Suppose $X$ has curvature $\geq k$ and $\gamma_{1}, \gamma_{2}$ are minimal in $X / G$ starting at a point $p(x)$. If $\gamma_{1}$ is extendable past $p(x)$, then for any lifts $\Gamma_{1}, \Gamma_{2}$ of $\gamma_{1}, \gamma_{2}$, respectively, at $x, \alpha\left(\gamma_{1}, \gamma_{2}\right)=\alpha\left(\Gamma_{1}, \Gamma_{2}\right)$.

Proof. Let $\gamma_{3}$ be minimal starting at $p(x)$ such that $\alpha\left(\gamma_{1}, \gamma_{3}\right)=\pi$ and let $\Gamma_{3}$ be a lift of $\gamma_{3}$ at $x$. Then $\alpha\left(\Gamma_{1}, \Gamma_{3}\right) \geq \alpha\left(\gamma_{1}, \gamma_{3}\right)=\pi$, so $\alpha\left(\Gamma_{1}, \Gamma_{3}\right)=\pi$. Then $\alpha\left(\gamma_{1}, \gamma_{2}\right) \leq \alpha\left(\Gamma_{1}, \Gamma_{2}\right)=\pi-\alpha\left(\Gamma_{2}, \Gamma_{3}\right) \leq \pi-\alpha\left(\gamma_{2}, \gamma_{3}\right)=\alpha\left(\gamma_{1}, \gamma_{2}\right)$.

Corollary 2.2. If $\gamma$ is minimal in $X / G$ and extendable past $p(x)$, then $\gamma$ has a unique lift at $x$.

Definition 2.3. Let $X$ be a space of curvature $\geq k, P$ be a connected subset of $X$, and $p \in P$. We say that $\gamma \in \bar{S}_{p}$ is tangent to $P$ if there exist $p_{i} \in P$ with $p_{i} \rightarrow p$ and $\gamma_{i}$ minimal from $p$ to $p_{i}$ such that $\lim _{i \rightarrow \infty} \alpha\left(\gamma, \gamma_{i}\right)=0$. We say that a curve $c:[-1,1] \rightarrow X$ such that $c(0)=p$ is tangent to $\gamma \in \bar{S}_{p}$ if $\lim _{t \rightarrow 0} \alpha\left(\gamma, \gamma_{t}\right)=0$, where $\gamma_{t}$ is any minimal curve from $p$ to $c(t)$.

We let $H_{x}$ denote the metric completion of the subset of $\bar{T}_{x}$ whose elements are scalar multiples of lifts of minimal curves in $X / G$. If $p(x)$ is not a geodesic terminal (i.e. every geodesic can be extended through it), $H_{x}$ is isometric, via the linear, lift-induced map, to the Hilbert space $\bar{T}_{p(x)}$. Let $V_{x}$ denote all elements of $\bar{T}_{x}$ orthogonal to $H_{x}$ (i.e., if $\gamma \in \bar{T}_{x}$ then for any $\left.\beta \in H_{x}, \alpha(\gamma, \beta)=\pi / 2\right)$. We let $S\left(H_{x}\right)=\bar{S}_{x} \cap H_{x}$ and $S\left(V_{x}\right)=\bar{S}_{x} \cap V_{x}$. Note that the geodesic directions are dense in $\bar{S}_{p(x)}$, and so also in $S\left(H_{x}\right)$.

Lemma 2.3. If $X / G$ is geodesically complete then if $\gamma$ is tangent to $G x$ at $x$, $\gamma \in S\left(V_{x}\right)$.

Proof. Suppose we have $g_{i} \rightarrow e$ and minimal curves $\gamma_{i}$ from $x$ to $g_{i} x$ such that $\lim \alpha\left(\gamma_{i}, \gamma\right)=0$. It suffices to prove that for any $\beta \in S\left(H_{x}\right), \lim \alpha\left(\gamma_{i}, \beta\right)=\pi / 2$. Since the geodesic directions are dense in $S\left(H_{x}\right)$ we can assume that $\beta$ is in fact a minimal geodesic. Since $G / X$ is geodesically complete we can find another geodesic $\beta^{\prime}$ such that $\alpha\left(\beta, \beta^{\prime}\right)=\pi$; we also assume $\beta$ and $\beta^{\prime}$ are minimal. If $p$ is the endpoint of $\beta$ then $d(p, x)=d(p, G x)$. Now if $\lim \inf \alpha\left(\gamma_{i}, \beta\right)=\pi / 2-\epsilon<\pi / 2$ then the curvature bound implies that $d\left(p, g_{i} x\right)<d(p, x)$ for some large $i$, a contradiction; hence $\lim \inf \alpha\left(\gamma_{i}, \beta\right) \geq \pi / 2$. Applying the same argument to $\beta^{\prime}$ we obtain $\lim \sup \alpha\left(\gamma_{i}, \beta\right) \leq \pi / 2$ from knowing $\alpha\left(\beta^{\prime}, \gamma_{i}\right)+\alpha\left(\gamma_{i}, \beta\right)=\pi$.

Recall that a space of curvature $\geq k$ is called almost Riemannian ([17]) if it is geodesically complete and finite dimensional. One of the important properties of an 
almost Riemannian space is that the space of directions at each point is compact. For the next proposition, recall that the strong excess of a triple $(b ; a, c)$ in a metric space is $\sigma(b ; a, c)=\frac{d(a, b)+d(b, c)-d(a, c)}{\min \{d(a, b), d(b, c)\}}$, which, for fixed $k$, is small if and only if the corresponding representative angle $\alpha_{k}(b ; a, c)$ in $S_{k}$ is close to $\pi$.

Proposition 2.4. If $X$ is geodesically complete and $X / G$ is almost Riemannian then $\gamma$ is tangent to $G x$ if and only if $\gamma \in S\left(V_{x}\right)$. In other words, the tangent space splits orthogonally into "tangent" and "lifted" components.

Proof. By the above lemma we need to show that if $\gamma \in S\left(V_{x}\right)$ then $\gamma$ is tangent to $G x$. Choose geodesic directions $\beta_{i}$ such that $\alpha\left(\beta_{i}, \gamma\right) \rightarrow 0$ and positive $t_{i} \rightarrow 0$ such the restriction of $\beta_{i}$ to $\left[-t_{i}, t_{i}\right]$ is strictly minimal. We claim that $d\left(\beta_{i}\left(t_{i}\right), G x\right) / t_{i} \rightarrow 0$. Suppose that there exists an $\epsilon>0$ such that for all sufficiently large $i,\left[d\left(\beta_{i}\left(t_{i}\right), G x\right) / t_{i}\right] \geq \epsilon$. If $p\left(\beta_{i}\left(t_{i}\right)\right)=p(x)$ for all large $i$, then $\beta_{i}\left(t_{i}\right) \in G x$, a contradiction, so $p\left(\beta_{i}\left(t_{i}\right)\right)$ and $p(x)$ are distinct. Let $\zeta_{i}$ be minimal in $X / G$ from $p(x)$ to $p\left(\beta_{i}\left(t_{i}\right)\right)$. Choosing a subsequence, if necessary $\left(\bar{S}_{p}\right.$ is compact), we can assume $\left\{\zeta_{i}\right\}$ is Cauchy. We will obtain a contradiction by showing that for any unit geodesic $\eta$ at $p(x), \limsup \alpha\left(\eta, \zeta_{i}\right) \leq \pi / 2$. Let $y_{i}=\eta\left(t_{i}\right), Y_{i}=\psi\left(t_{i}\right)$, where $\psi$ is a lift of $\eta$ at $x$. Finally, let $d_{i}=d\left(y_{i}, p\left(\beta_{i}\left(t_{i}\right)\right)\right.$ and $D_{i}=d\left(Y_{i}, \beta_{i}\left(t_{i}\right)\right)$. By Lemma 1.3 , [17], since $\beta_{i}$ is Cauchy, $\lim _{i \rightarrow \infty}\left(D_{i}-\sqrt{2} t_{i}\right) / t_{i}=0$. Using the distance decreasing property of $p$, we have

$$
\begin{aligned}
\lim _{i \rightarrow \infty} & \sup \left[\left(d_{i}-\sqrt{2} t_{i}\right) / d\left(p(x), p\left(\beta_{i}\left(t_{i}\right)\right)\right]\right. \\
& \leq \lim _{i \rightarrow \infty} \sup \left[\left(D_{i}-\sqrt{2} t_{i}\right) / d\left(\beta_{i}\left(t_{i}\right), G x\right)\right] \\
& \leq\left|\lim _{i \rightarrow \infty} \sup \left[\left(D_{i}-\sqrt{2} t_{i}\right) / d\left(\beta_{i}(t), G x\right)\right]\right| \\
& \leq \frac{1}{\epsilon}\left|\lim \sup \left[\left(D_{i}-\sqrt{2} t_{i}\right) / t_{i}\right]\right|=0 .
\end{aligned}
$$

Lemma 1.3, [17] now implies that $\limsup _{i \rightarrow \infty} \alpha\left(\eta, \zeta_{i}\right) \leq \pi / 2$.

To complete the proof of the proposition, suppose that $\delta_{i}:=d\left(\beta_{i}\left(t_{i}\right), G x\right)$ is realized by $d\left(\beta_{i}\left(t_{i}\right), g_{i} x\right)$ and let $m_{i}=\min \left\{t_{i}=d\left(\beta_{i}\left(t_{i}\right), x\right), d\left(x, g_{i} x\right)\right\}$. Then the triangle inequality implies that

$$
t_{i}+d\left(x, g_{i} x\right)-d\left(\beta_{i}\left(-t_{i}\right), g_{i} x\right) / m_{i} \leq\left(2 t_{i}+\delta_{i}-2 t_{i}+\delta_{i}\right) / m_{i}=2 \delta_{i} / m_{i} .
$$

Now $m_{i} \leq t_{i} \leq m_{i}+\delta_{i}$, so $t_{i} / m_{i} \rightarrow 1$ and $\lim \delta_{i} / m_{i}=\lim \left(\delta_{i} / t_{i}\right)\left(t_{i} / m_{i}\right)=0$. In other words, $\sigma\left(x ; g_{i} x, \beta_{i}\left(-t_{i}\right)\right)$ tends to zero, so if $\tau_{i}$ is minimal from $x$ to $g_{i} x$ then $\alpha\left(-\beta_{i}, \tau_{i}\right) \rightarrow \pi$ and $\lim \alpha\left(\gamma, \tau_{i}\right)=\lim \alpha\left(\beta_{i}, \tau_{i}\right)=0$.

Remark 2.1. The above results are valid when replacing "extendable" by "almost extendable" ([21]). For example, in this way it is possible to find "good" orbits at which lifts are angle preserving, by taking orbits whose tangent space in the quotient is Euclidean. However, we will not use these facts in this paper.

\section{Products}

To prove our main theorem we need to consider products of countably infinitely many factors. The key to understanding countable products is to regard elements in any tangent cone of a factor not as unit parameterized geodesics of various lengths 
(which is the interpretation suggested by the exponential map), but as constantmultiple reparameterizations of the corresponding unit geodesics.

If $\left(X, d_{1}\right)$ and $\left(Y, d_{2}\right)$ are inner metric spaces, the product of $X$ and $Y$ is the space $\left(X \times Y, d_{1} \times d_{2}\right)$, where $d_{1} \times d_{2}$ is the metric given by $d_{1} \times d_{2}\left[\left(x_{1}, y_{1}\right),\left(x_{2}, y_{2}\right)\right]=$ $\sqrt{d_{1}\left(x_{1}, x_{2}\right)^{2}+d_{2}\left(y_{1}, y_{2}\right)^{2}}$. It is not hard to verify that $d_{1} \times d_{2}$ is an inner metric. Minimal curves in $X \times Y$ are precisely curves of the form $(\gamma(t), \alpha(t))$ where $\gamma$ and $\alpha$ are minimal or constant in $X$ and $Y$, respectively, and $L(\gamma)^{2}+L(\alpha)^{2}=L(\gamma, \alpha)^{2}$. When no confusion will result, we denote the metric on the factors and the product all by $d$.

Lemma 3.1. If $(\gamma, \beta)$ is a minimal geodesic in $X \times Y$ from $(x, y)$ to $(w, z)$, with $\gamma, \beta$ non-constant, then the triangle made by the curves $(\gamma, \beta),(\gamma, y)$, and $(w, \beta)$ embeds isometrically in the plane. In particular, the angle $\alpha((\gamma, \beta),(\gamma, y))$ between the geodesic and its projection onto $X \times y$ exists and is equal to $\tan ^{-1}\left(\frac{L(\beta)}{L(\gamma)}\right)$.

Proof.

$$
d((x, y),(\gamma(t), y))^{2}+d((\gamma(t), y)),(\gamma(t), \beta(t))^{2}=d((x, y),(\gamma(t), \beta(t)))^{2} .
$$

Since $(\gamma, \beta)$ is proportional to arclength, this equation can be rewritten as

$$
(t \cdot L(\gamma))^{2}+(t \cdot L(\beta))^{2}=(t \cdot L(\gamma, \beta))^{2}
$$

i.e. representatives of the triple $((x, y) ;(\gamma(t), y),(\gamma(t), \beta(t)))$ in the plane are vertices of triangles similar to the right triangle having legs of lengths $L(\gamma)$ and $L(\beta)$. In other words, for all $t$, the representative angles are the same, namely, $\tan ^{-1}\left(\frac{L(\beta)}{L(\gamma)}\right)$.

If $\gamma$ is a unit geodesic in $X$, we denote by $k \gamma$ the curve defined by $k \gamma(t)=\gamma(k t)$. If $\beta$ is a unit geodesic in $Y$, we denote by $k \gamma+m \beta$ the curve $(k \gamma, m \beta)$ in $X \times Y$, which is either a constant map $(k=m=0)$ or a geodesic; by Lemma $3.1, k \gamma+m \beta$ is a unit geodesic if and only if $k^{2}+m^{2}=1$. When we use this notation it will always be assumed that $\gamma$ and $\beta$ are unit parameterized and $m, k \geq 0$. Every unit geodesic $\left(c_{1}, c_{2}\right)$ in $X \times Y$ can be written in this form (if one factor, say $c_{1}$, is constant, we pick a geodesic $\gamma_{1}$ in $X$ arbitrarily and let the coefficient be 0 ). The expression of a unit geodesic in the form $k \gamma+m \beta$ is unique if one ignores terms with 0 as coefficient. In what follows, $k_{i}=0$, we define $c_{p_{i}}(\gamma) / k_{i}$ to be $\infty$. (Recall that $c_{p}$ denotes the cut radius at $p$, cf. Section 1$)$.

Lemma 3.2. For any $\left(p_{1}, p_{2}\right) \in X_{1} \times X_{2}$ and minimal $\gamma=k_{1} \gamma_{1}+k_{2} \gamma_{2}$, with $k_{1}^{2}+k_{2}^{2}=1$, starting at $p=\left(p_{1}, p_{2}\right), c_{p}(\gamma)=\min \left\{c_{p_{i}}\left(\gamma_{i}\right) / k_{i}\right\}$.

Proof. Let $c=\min \left\{c_{p_{i}}\left(\gamma_{i}\right) / k_{i}\right\}$. Then each curve $k_{i} \gamma_{i}$ is minimal on $[0, c]$, so $\gamma$ is also minimal on $[0, c]$, and $c_{p}(\gamma) \geq c$. Conversely, $\gamma$ is minimal on $\left[0, c_{p}(\gamma)\right]$ so each $k_{i} \gamma_{i}$ is minimal on this interval. But then $c_{p_{i}}\left(\gamma_{i}\right) \geq k_{i} c_{p}(\gamma)$ for both $i$ and $c \geq c_{p}(\gamma)$.

If $X$ and $Y$ are Riemannian manifolds, $d_{1} \times d_{2}$ coincides with the distance induced by the usual product Riemannian metric on $X \times Y$. From this last fact it follows that if $X$ and $Y$ are inner metric spaces of curvature $\geq k$ and $\geq k^{\prime}$, respectively, then any four points in $X \times Y$ can be isometrically embedded in $S_{k} \times S_{k^{\prime}}$. By the definition of bounded curvature this means that $X \times Y$ has curvature $\geq \min \left\{0, k, k^{\prime}\right\}$ (cf. also [7]). In a similar way, we see that angles in $X \times Y$ are measured in the same way as angles in a product of Riemannian manifolds. In other words: 
Lemma 3.3. Suppose $X$ and $Y$ have curvature $\geq k$ and $\gamma=k_{1} \gamma_{1}+k_{2} \gamma_{2}$ and $\beta=m_{1} \beta_{1}+m_{2} \beta_{2}$ are unit minimal starting at a common point in $X \times Y$. Then $\cos \alpha(\gamma, \beta)=k_{1} m_{1} \cos \alpha\left(\gamma_{1}, \beta_{1}\right)+k_{2} m_{2} \cos \alpha\left(\gamma_{2}, \beta_{2}\right)$. In particular, $\alpha(\gamma, \beta) \geq$ $\alpha\left(\gamma_{i}, \beta_{i}\right)$ for all $i$ such that $k_{i}, m_{i} \neq 0$.

Proposition 3.4. For any $\epsilon>0$ and $k$, there exists a $\delta>0$ depending only $k$ such that if $X$ and $Y$ have curvatuare $\geq k$, and $\gamma=k_{1} \gamma_{1}+k_{2} \gamma_{2}$ and $\beta=m_{1} \beta_{1}+m_{2} \beta_{2}$ are unit minimal in $X \times Y$ such that $\alpha(\gamma, \beta)<\delta$ then $\left|k_{i}-m_{i}\right|<\epsilon$.

Proof. Let $k^{\prime}=\min \{k, 0\}$. Fix $\delta>0$ small enough that if $\Gamma_{1}$ and $\Gamma_{2}$ are unit minimal in $S_{k^{\prime}}$ such that if $\alpha\left(\Gamma_{1}, \Gamma_{2}\right)<\delta$ then for any $t<1, d\left(\Gamma_{1}(t), \Gamma_{2}(t)\right) / t<\epsilon / 2$. Assume first that $k_{i}, m_{i}>0$. Then for any positive $t<\min _{i}\left\{c_{p_{i}}\left(\gamma_{i}\right) / m_{i}, c_{p_{i}}\left(\gamma_{i}\right) / k_{i}\right\}$, if $\alpha(\gamma, \beta)<\delta$ then by Lemma 3.3, $\alpha\left(\gamma_{i}, \beta_{i}\right)<\delta$ and

$$
\begin{gathered}
\left|k_{i}-m_{i}\right|=\left(\frac{1}{t}\right)\left|k_{i} t-m_{i} t\right|=\frac{1}{t} d\left(\gamma_{i}\left(k_{i} t\right), \gamma_{i}\left(m_{i} t\right)\right) \\
\leq \frac{1}{t}\left(d\left(\gamma_{i}\left(k_{i} t\right), \beta_{i}\left(m_{i} t\right)\right)+d\left(\beta_{i}\left(m_{i} t\right), \gamma_{i}\left(m_{i} t\right)\right)\right) \\
<\frac{1}{t} d(\gamma(t), \beta(t))+\epsilon / 2<\epsilon .
\end{gathered}
$$

If, say, $m_{i}>0$ and $k_{i}=0$ then $m_{i}=\frac{d\left(\beta_{i}\left(m_{i} t\right), p_{i}\right)}{t}=\frac{d\left(\beta_{i}\left(m_{i} t\right), \gamma_{i}\left(k_{i} t\right)\right)}{t} \leq$ $\frac{d(\beta(t), \gamma(t))}{t}<\epsilon / 2$. If, say, $m_{1}=k_{1}=0$ then $\gamma$ and $\beta$ lie in a single $Y$ slice, and the proof is immediate from our choice of $\delta$. The other cases are essentially the same.

Since $\bar{S}_{p}$ is by definition the space of equivalence classes of Cauchy sequences in $S_{p}$, we immediately have the following corollaries of Lemma 3.3 and Proposition 3.4. Note that we identify $k_{1} \gamma_{1}+k_{2} \gamma_{2}$ with $k_{1}^{\prime} \gamma_{1}^{\prime}+k_{2} \gamma_{2}$ if $k_{1}=k_{1}^{\prime}=0$, and let $-k \gamma(t)=\gamma(-k t)$ whenever this makes sense (i.e. $\gamma$ extends past $\gamma(0)$ ). To avoid technicalities later, if $k_{i}=0$, we will always assume that $\gamma_{i}$ is a geodesic direction (we can, of course, always choose it this way).

Corollary 3.5. For any $p_{1} \in X$ and $p_{2} \in Y, \bar{S}_{\left(p_{1}, p_{2}\right)}=\left\{k_{1} \gamma_{1}+k_{2} \gamma_{2}: k_{1}^{2}+k_{2}^{2}=1\right.$, $k_{i} \geq 0$, and $\left.\gamma_{i} \in \bar{S}_{p_{i}}\right\}$, and $c_{\left(p_{1}, p_{2}\right)}(\gamma)=\min \left\{c_{p_{i}}\left(\gamma_{i}\right) / k_{i}\right\}$.

Corollary 3.6. If $X$ and $Y$ are geodesically complete then for all $p=\left(p_{1}, p_{2}\right) \in$ $X \times Y, \bar{T}_{p}=\bar{T}_{p_{1}} \times \bar{T}_{p_{2}}$ (as Hilbert spaces).

The above constructions are easily extended to any finite number of factors by simple inductive arguments; the details are left to the reader. We only verify the following:

Lemma 3.7. If each $X_{i}$ has curvature $\geq k$ and $\gamma=\sum_{i=1}^{k} k_{i} \gamma_{i}$ is a unit geodesic then $c_{p}(\gamma)=\min \left\{c_{p_{i}}\left(\gamma_{i}\right) / k_{i}\right\}$.

Proof. The case $k=2$ is Lemma 3.2; we proceed by induction. Suppose the lemma holds for $k-1>1$. If any $k_{i}=0$ we can eliminate a factor and apply the induction hypothesis, so we suppose that $k_{i}>0$ for all $i$. By re-indexing the product, if necessary, we can suppose that

$$
c_{p_{k}}\left(\gamma_{k}\right) / k_{k} \leq c_{p_{k-1}}\left(\gamma_{k-1}\right) / k_{k-1} \leq \cdots \leq c_{p_{1}}\left(\gamma_{1}\right) / k_{1} .
$$


Write $\sum_{i=1}^{k} k_{i} \gamma_{i}=k_{k} \gamma_{k}+\sum_{i=1}^{k-1} k_{i} \gamma_{i}$. By the induction hypothesis,

$$
c_{\left(p_{1} \ldots, p_{k-1}\right)}\left(\sum_{i=1}^{k-1} k_{i} \gamma_{i}\right)=\frac{c_{p_{k}}\left(\gamma_{k-1}\right)\left(\sum_{i=1}^{k-1} k_{i}^{2}\right)^{1 / 2}}{k_{k-1}} .
$$

Now

$$
c_{p_{k}}\left(\gamma_{k}\right) / k_{k} \leq c_{p_{k-1}}\left(\gamma_{k-1}\right) / k_{k-1}=\frac{c_{\left(p_{1}, \ldots, p_{k-1}\right)}\left(\sum_{i=1}^{k-1} k_{i} \gamma_{i}\right)}{\left(\sum_{i=1}^{k-1} k_{i}^{2}\right)^{1 / 2}}
$$

and the proof is complete by Lemma 3.2.

Now suppose $\left\{X_{i}, d_{i}\right\}$ is a countable collection of inner metric spaces such that $\left\{\operatorname{diam}\left(X_{i}\right)\right\}$ is square summable. We define a metric on $X=\prod_{i=1}^{\infty} X_{i}$ in the following way. Choose a fixed element $\left(x_{1}, x_{2}, \ldots\right)$ of $X$. For each $j$, let $X^{j}$ denote $\prod_{i=1}^{j} X_{i}$. Then $X^{j}$ isometrically embeds in $X^{j+1}$ via the mapping $\left(y_{1}, \ldots, y_{j}\right) \mapsto$ $\left(y_{1}, \ldots, y_{j}, x_{j+1}\right)$, and with this embedding, $d_{H}\left(X^{j}, X^{j+1}\right) \leq \operatorname{diam}\left(X_{j+1}\right)$. Thus the sequence $\left\{X^{j}\right\}$ is Cauchy in the Gromov-Hausdorff metric ([10]), and the unique limit of this sequence is identified with $X$. It is easy to see that this definition is equivalent to setting

$$
\begin{aligned}
& d\left(\left(y_{1}, y_{2}, \ldots\right),\left(z_{1}, z_{2}, \ldots\right)\right) \\
& \quad=\lim _{j \rightarrow \infty} d_{1} \times \ldots \times d_{j}\left(\left(y_{1}, \ldots, y_{j}\right),\left(z_{1}, \ldots, z_{j}\right)\right)=\sqrt{\sum d_{i}\left(y_{i}, z_{i}\right)^{2}} .
\end{aligned}
$$

The topology induced by this metric is equivalent to the usual product topology, and so $X$ is a compact inner metric space if each $X_{i}$ is compact. Also, it is immediate from the definition that for any $j, X$ is isometric to $\prod_{i=1}^{j} X_{i} \times \prod_{i=j}^{\infty} X_{i}$. Each finite product of the factors $X_{i}$ is embedded naturally in $X$ by fixing all the remaining coordinates. We recall the following definition (cf. [21]):

Definition 3.1. A subset $Y$ of an inner metric space $X$ is called metrically embedded if the induced metric and the induced inner metric coincide on $Y$.

When $Y$ is metrically embedded in $X$, every minimal curve between points $x, y \in$ $Y$ is also a minimal curve in $X$. Consequently, if $X$ has curvature $\geq k$ then so does $Y$, and the angle between minimal curves $\gamma$ and $\beta$ in $Y$ (if it exists) is the same as the angle in $X$. In other words, for any point $p \in Y$, the inclusion of $\bar{S}_{p} Y$ in $\bar{S}_{p} X$ is an isometry, and $\bar{S}_{p} Y$ is a metrically embedded subset of $\bar{S}_{p} X$ if both are inner metric spaces.

It is immediate from the definition of the product metric that each $X_{i}$ is metrically embedded in $X$. If each $X_{i}$ has curvature $\geq k$ then $X$ has curvature $\geq \min \{0, k\}$, since we have expressed it above as a Gromov-Hausdorff limit. If $\left(c_{1}, c_{2}, \ldots\right):[0,1] \rightarrow X$ is minimal then each $c_{i}$ is minimal in $X_{i}$. In fact, we need only show that if we set $c_{i}(0)=x_{i}, c_{i}(1 / 2)=y_{i}$, and $c_{i}(1)=z_{i}$ then $y_{i}$ is a midpoint between $x_{i}$ and $z_{i}$. However, the Minkowski and triangle inequalities imply that $\left(\operatorname{setting} x=\left(x_{1}, \ldots\right)\right.$, etc.) $d(x, z)=d(x, y)+d(y, z)=\sqrt{\sum d\left(x_{i}, y_{i}\right)^{2}}+$ 
$\sqrt{\sum d\left(y_{i}, z_{i}\right)^{2}} \geq \sqrt{\sum\left(d\left(x_{i}, y_{i}\right)+d\left(y_{i}, z_{i}\right)\right)^{2}} \geq \sqrt{\sum d\left(x_{i}, z_{i}\right)^{2}}=d(x, z)$. If $y_{i}$ were not a midpoint for some $i$ then the second (triangle) inequality would be a strict inequality, a contradiction.

Proposition 3.8. Let $c_{i}$ be a unit parameterized curve in $X_{i}$. Then for $k_{i} \geq 0$, a curve $c(t)=\left(c_{1}\left(k_{1} t\right), c_{2}\left(k_{2} t\right), \ldots\right)$ in $X=\prod_{i=1}^{\infty} X_{i}$ is rectifiable if and only if $\sum_{i=1}^{\infty} k_{i}^{2}<$ $\infty$ (and $c$ is unit parameterized if and only if $\left.\sum_{i=1}^{\infty} k_{i}^{2}=1\right)$.

Proof. It suffices to assume $c$ is defined on a fixed closed interval $[a, b]$. For any $s<t$

$$
\begin{gathered}
d(c(s), c(t))^{2}=\sum_{i=1}^{\infty} d\left(c_{i}\left(k_{i} s\right), c_{i}\left(k_{i} t\right)\right)^{2} \\
\leq \sum_{i=1}^{\infty}\left|k_{i} t-k_{i} s\right|^{2}=\sum_{i=1}^{\infty} k_{i}^{2}|t-s|^{2} .
\end{gathered}
$$

If $\sum_{i=1}^{\infty} k_{i}^{2}=K^{2}<\infty$ then we have that $d(c(s), c(t)) \leq K(t-s)$; i.e. $c$ is rectifiable and $L\left(\left.c\right|_{[a, b]}\right) \leq K(b-a)$. Now for any $\epsilon>0$ we can find $M$ such that $\sum_{i=M}^{\infty} k_{i}^{2}(b-a)^{2}<\epsilon$. Choose a partition $a=s_{1}<\ldots<s_{k}=b$ such that for all $i<M, k_{i}^{2}(b-a)^{2}-\sum_{j=1}^{k-1} d\left(c_{i}\left(k_{i} s_{j}\right), c_{i}\left(k_{i} s_{j+1}\right)\right)^{2}<\epsilon / M$. Then

$$
\begin{aligned}
L(c)^{2} & \geq\left(\sum_{j=1}^{k-1} d\left(c\left(s_{j}\right), c\left(s_{j+1}\right)\right)\right)^{2} \geq \sum_{j=1}^{k-1} \sum_{i=1}^{\infty} d\left(c_{i}\left(s_{j}\right), c_{i}\left(s_{j+1}\right)\right)^{2} \\
& \geq \sum_{j=1}^{k-1} \sum_{i=1}^{M} d\left(c_{i}\left(k_{i} s_{j}\right), c_{i}\left(k_{i} s_{j+1}\right)\right)^{2}>\sum_{i=1}^{M} k_{i}^{2}(b-a)^{2}-\epsilon>K^{2}(b-a)^{2}-2 \epsilon .
\end{aligned}
$$

In other words, $L(c)=K(b-a)$, and $c$ is unit parameterized if and only if $K=1$.

Now suppose $\sum_{i=1}^{\infty} k_{i}^{2}=\infty$. For any $N$ fix $M$ such that $\sum_{i=1}^{M} k_{i}^{2}>N$ and choose a partition $a=s_{1}<\ldots<s_{k}=b$ such that for all $i<M, k_{i}^{2}(b-a)^{2}-$ $\sum_{j=1}^{k-1} d\left(c_{i}\left(k_{i} s_{j}\right), c_{i}\left(k_{i} s_{j+1}\right)\right)^{2}<1 / M$. Then

$$
\begin{gathered}
\left(\sum_{j=1}^{k-1} d\left(c\left(k_{i} s_{j}\right), c\left(k_{i} s_{j+1}\right)\right)\right)^{2}=\sum_{j=1}^{k-1} \sum_{i=1}^{\infty} d\left(c_{i}\left(k_{i} s_{j}\right), c_{i}\left(k_{i} s_{j+1}\right)\right)^{2} \\
>\sum_{j=1}^{k-1} \sum_{i=1}^{M} d\left(c_{i}\left(k_{i} s_{j}\right), c_{i}\left(k_{i} s_{j+1}\right)\right)^{2} \\
>\sum_{i=1}^{M}\left(k_{i}^{2}(b-a)^{2}-1 / M\right)>N(b-a)^{2}-1 .
\end{gathered}
$$


Corollary 3.9. Every unit parameterized geodesic in $X=\prod_{i=1}^{\infty} X_{i}$ can be written uniquely (except for $\gamma_{i}$ when $k_{i}=0$ ) in the form

$$
\sum_{i=1}^{\infty} k_{i} \gamma_{i}(t)=\left(\gamma_{1}\left(k_{1} t\right), \gamma_{2}\left(k_{2} t\right), \ldots\right)
$$

where each $\gamma_{i}$ is a unit geodesic in $X_{i}, k_{i} \geq 0$, and $\sum_{i=1}^{\infty} k_{i}^{2}=1$.

As in the case of finitely many factors, when we use the notation $\sum_{i=1}^{\infty} k_{i} \gamma_{i}$, it will always be assumed that $k_{i} \geq 0$ and $\gamma_{i}$ is unit. From now on we assume that $X=\prod_{i=1}^{\infty} X_{i}$ and that each $X_{i}$ has curvature $\geq k$. In general, continuity of the angle fails with Gromov-Hausdorff limits: the angle of the limit of curves can be strictly smaller than the limit of the angles. In the present case, however, this does not occur. For the purposes of the next proposition, we take the angle between any geodesic and a constant map to be 0 .

Proposition 3.10. Suppose $\gamma=\left(\gamma_{1}, \ldots\right)$ and $\beta=\left(\beta_{1}, \ldots\right)$ are geodesics starting at a common point in $X$. Then $\alpha(\gamma, \beta)$ is the limit of the increasing sequence $\alpha\left(\left(\gamma_{1}, \ldots, \gamma_{k}\right),\left(\beta_{1}, \ldots, \beta_{k}\right)\right)$.

Proof. The sequence is increasing by Lemma 3.3. Since we are only interested in the angle, we can assume that both $\gamma$ and $\beta$ are minimal and have the same length $L>0$. For any $i$, split $X$ as the product $\prod_{k=1}^{i} X_{k} \times \prod_{k=i+1}^{\infty} X_{k}$. Observe that $L\left(\gamma_{k}\right), L\left(\beta_{k}\right)<d_{k}:=\operatorname{diam}\left(X_{k}\right)$. Set $\gamma^{i}=\left(\gamma_{1}, \ldots, \gamma_{i}\right), \beta^{i}=\left(\beta_{1}, \ldots, \beta_{i}\right)$ and identify $\gamma^{i}, \beta^{i}$ with the geodesics $\gamma^{i} \times(e, e, \ldots)$ and $\beta^{i} \times(e, e, \ldots)$ in the slice $\prod_{k=1}^{i} X_{k}$. According to Lemma 3.1 and the triangle inequality, this means that $\alpha\left(\gamma^{i}, \gamma\right)$ and $\alpha\left(\beta^{i}, \beta\right)$ are both smaller than $\tan ^{-1}\left(\frac{\sum_{k=i}^{\infty} d_{k}^{2}}{L_{i}}\right)$, where $L_{i}=\min \left\{L\left(\gamma^{i}\right), L\left(\beta^{i}\right)\right\}$ and tends to $L$ as $i$ becomes large. In other words, both $\alpha\left(\gamma^{i}, \gamma\right)$ and $\alpha\left(\beta^{i}, \beta\right)$ tend to 0 as $i$ becomes large.

We wish to extend Corollary 3.9 to any direction in $X$. Since directions may not be uniquely associated with curves, our statement is neccessarily about limits. What we are essentially doing, however, is identifying $\bar{S}_{p}$ with $\left\{\sum_{i=1}^{\infty} k_{i} \gamma_{i}: \sum_{i=1}^{\infty} k_{i}^{2}=\right.$ $\left.1, \gamma_{i} \in \bar{S}_{p_{i}}\right\}$

Proposition 3.11. Let $p \in X$ and $\gamma \in \bar{S}_{p}$. Then there are unique $k_{i} \geq 0$ with $\sum_{i=1}^{\infty} k_{i}^{2}=1$ and unique $\left(\right.$ for $k_{i} \neq 0$ ) directions $\gamma_{i} \in \bar{S}_{p_{i}}$ so that $c_{p}(\gamma)=$ $\inf \left\{c_{p_{i}}\left(\gamma_{i}\right) / k_{i}\right\}$, and for any geodesic directions $\beta_{j}=\sum_{i=1}^{\infty} k_{i j} \beta_{i j} \in S_{p}$ such that $\alpha\left(\beta_{j}, \gamma\right) \rightarrow 0, \lim _{j \rightarrow \infty} k_{i j}=k_{i}$ and $\lim _{j \rightarrow \infty} \beta_{i j}=\gamma_{i}$ when $k_{i} \neq 0$. Conversely, suppose we are given directions $\gamma_{i}$ and $k_{i} \geq 0$ such that $\sum_{i=1}^{\infty} k_{i}^{2}=1$. Then for any geodesic 
directions $\beta_{i j}$ and $k_{k j} \geq 0$ such that $\sum_{i=1}^{\infty} k_{i j}^{2}=1$ and $\alpha\left(\beta_{i j}, \gamma_{i}\right) \rightarrow 0$ for all $i$ such that $k_{i} \neq 0$, the geodesics $\beta^{k}:=\sum_{i=1}^{k} k_{i k} \beta_{i k}$ form a Cauchy sequence.

Proof. Every unit geodesic $\gamma$ in $X$ is uniquely represented in the form $\gamma=\sum_{i=1}^{\infty} k_{i} \gamma_{i}$, with non-negative coefficients square summable to 1 . By the semicontinuity of the cut radius map, $c_{p}(\gamma) \geq \inf \left\{c_{p_{i}}\left(\gamma_{i}\right) / k_{i}\right\}$. On the other hand, writing

$$
\gamma=\sum_{i=1}^{k} \frac{k_{i}}{\left(\sum_{i=1}^{k} k_{i}^{2}\right)^{1 / 2}} \gamma_{i}+\sum_{i=k+1}^{\infty} \frac{k_{i}}{\left(\sum_{i=k+1}^{\infty} k_{i}^{2}\right)^{1 / 2}} \gamma_{i}
$$

we see that

$$
c_{p}(\gamma) \leq \inf _{i \leq k}\left\{\left(\sum_{i=1}^{k} k_{i}^{2}\right)^{1 / 2} c_{p_{i}}\left(\gamma_{i}\right) / k_{i}\right\} \rightarrow \inf \left\{c_{p_{i}}\left(\gamma_{i}\right) / k_{i}\right\},
$$

so $c_{p}(\gamma)=\inf \left\{c_{p_{i}}\left(\gamma_{i}\right) / k_{i}\right\}$.

If $\gamma$ is not a geodesic direction (i.e. $c_{p}(\gamma)=0$ ), choose $\beta_{j} \in S_{p}$ such that $\beta_{j} \rightarrow \gamma$, where $\beta_{j}=\sum_{i=1}^{\infty} k_{i j} \beta_{i j}$. By Lemma 3.3 and Proposition 3.4, for fixed $k$ the sequence $\beta_{j}^{k}=\sum_{i=1}^{k} k_{i j} \beta_{i j}$ is Cauchy with limit $\gamma^{k}=\sum_{i=1}^{k} k_{i} \gamma_{i}$, where the coefficients $k_{i}=\lim _{j \rightarrow \infty} k_{i j}$ satisfy $\sum_{i=1}^{\infty} k_{i}^{2}=1$. Now for $j$ sufficiently large, $\alpha\left(\gamma^{k}, \beta_{j}^{k}\right)$ is small. Also, if $s_{j k}:=\sum_{i=k}^{\infty} k_{i j}$, then for $j, k$ sufficently large, $s_{j k}$ is small. In other words, for $j, k$ sufficiently large, $\alpha\left(\beta_{j}, \beta_{j}^{k}\right)$ is small; that is, $\gamma^{k} \rightarrow \gamma$ in $\bar{S}_{p}$, and we have identified $\gamma$ with $\sum_{i=1}^{\infty} k_{i} \gamma_{i}$. It now follows that $\inf \left\{c_{p_{i}}\left(\gamma_{i}\right) / k_{i}\right\}=0$ by the semicontinuity of the cut radius. Thus we have associated to each direction a sum $\sum_{i=1}^{\infty} k_{i} \gamma_{i}$ and verified the cut radius formula. Our choice of $\beta_{j}$ was arbitrary and we proved convergence without taking a subsequence. From this it follows that the sum $\sum_{i=1}^{\infty} k_{i} \gamma_{i}$ is unique.

The converse statement is an immediate consequence of Lemma 3.3.

If $Y$ is a geodesically complete space of curvature bounded below then the inner product induced on the tangent space is completely determined by angles and lengths of curves; hence we have:

Corollary 3.12. If each $X_{i}$ is geodesically complete, then for any $p \in X, \bar{T}_{p}$ is identified with the Hilbert space of formal sums $\sum_{i=1}^{\infty} k_{i} \gamma_{i}$, where each $\gamma_{i}$ is unit minimal in $X_{i}, \sum_{i=1}^{\infty} k_{i}^{2}<\infty$, and the inner product is given by

$$
\begin{aligned}
\left\langle\sum_{i=1}^{\infty} k_{i} \gamma_{i}, \sum_{i=1}^{\infty} m_{i} \beta_{i}\right\rangle & =\lim _{k \rightarrow \infty}\left\langle\sum_{i=1}^{k} k_{i} \gamma_{i}, \sum_{i=1}^{k} m_{i} \beta_{i}\right\rangle \\
& =\lim _{k \rightarrow \infty} \sum_{i=1}^{k} k_{i} m_{i}\left\langle\gamma_{i}, \beta_{i}\right\rangle .
\end{aligned}
$$


Note that $X$ need not be geodesically complete in general, although this requires a certain amount of asymmetry from the cut radius at each $p_{i}$.

Proposition 3.13. If each $X_{i}$ has positive cut radius, then $X$ is weakly convex and for each direction $\gamma=\sum_{i=1}^{\infty} k_{i} \gamma_{i}$, the corresponding unit curve $c=\left(k_{1} \gamma_{1}, k_{2} \gamma_{2}, \ldots\right)$ is tangent to $\gamma$.

Proof. For any $p \in X$, let $W_{k}$ be the subspace of $\bar{T}_{p}$ consisting of all geodesics $\sum_{i=1}^{k} k_{i} \gamma_{i}$. Since $X_{i}$ has positive cut radius, the fact that $W_{p}=\bigcup_{k=1}^{\infty} w_{k}$ satisfies the requirements of Definition 1.2 follows easily from Proposition 3.11. Since each $X_{i}$ has positive cut radius, each $\gamma_{i}$ is a bona fide geodesic, so $c$ is in fact a curve. We need to show that $c$ is tangent to $\gamma$. Suppose that $\beta_{t}=\sum_{i=1}^{\infty} m_{t i} \beta_{t i}$ is minimal from $c(0)$ to $c(t)$ parameterized so that $\beta_{t}(t)=c(t)$. Then each $\beta_{t}^{k}=\sum_{i=1}^{k} m_{t i} \beta_{t i}$ is minimal from $\gamma^{k}(0)$ to $\gamma^{k}(t)$, where $\gamma^{k}=\sum_{i=1}^{k} k_{i} \gamma_{i}$. As soon as $t<\min _{i \leq k}\left\{c_{p_{i}}\left(\gamma_{i}\right) / k_{i}\right\}$, $\beta_{t}^{k}$ must coincide with $\gamma^{k}$ (even up to parameterization, since $\beta_{t}^{k}(t)=\gamma^{k}(t)$ ), i.e. $m_{i t}=k_{i}$. Now $\sum_{i=1}^{k} k_{i}^{2}=\sum_{i=1}^{k} m_{t i}^{2} \leq \sum_{i=1}^{\infty} m_{t i}^{2}=d(c(t), c(0))^{2} / t^{2} \leq \sum_{i=1}^{\infty} k_{i}^{2}=1$. In other words, by Lemma 3.1, for $k$ fixed and small $t, \alpha\left(\beta_{t}^{k}, \beta_{t}\right)$ is uniformly small. For any $\epsilon>0$ there exists a $k$ such that $\alpha\left(\gamma^{k}, \gamma\right)<\epsilon$, so there exists a $t>0$ such that $\alpha\left(\beta_{t}^{k}, \gamma\right)<\epsilon$ and hence $\alpha\left(\beta_{t}, \gamma\right)<\epsilon$.

\section{Bi-InVARIant Inner Metrics}

The Gleason-Iwasawa-Yamabe theory of locally compact groups (cf. [9]) implies that a connected locally compact group splits locally as a product between a Lie group and a compact group. This theorem essentially reduces our results on locally compact groups to results on compact groups. For compact groups it is important to consider metrics which are bi-invariant; such metrics are also very interesting in their own right.

Lemma 4.1. A metric on a group $G$ is bi-invariant if and only if it is invariant and the inverse map $a \rightarrow a^{-1}$ is an isometry.

Proof. If $G$ has a bi-invariant metric then $d(a, b)=d\left(e, a^{-1} b\right)=d\left(b^{-1}, a^{-1}\right)$. Conversely, if the metric is invariant and the inverse is an isometry, $d(a b, c b)=$ $d\left(b^{-1} a^{-1}, b^{-1} c^{-1}\right)=d\left(a^{-1}, c^{-1}\right)=d(a, c)$.

Proposition 4.2. If $G$ is a group with a bi-invariant inner metric of curvature bounded below then every geodesic $\gamma$ through e is a homomorphism (i.e. the image of the curve is a 1-parameter subgroup).

Proof. Assume $\gamma$ is unit parameterized. We will show first that $\gamma(-t)=\gamma(t)^{-1}$. Since the inverse mapping is an isometry, the curve $\gamma(t)^{-1}$ is a geodesic. Consider the geodesic $\beta_{s}(t)=\gamma(s)^{-1} \gamma(t)$ for fixed $s$, which has the property that $\beta_{s}(s)=e$ and $\beta_{s}(0)=\gamma(s)^{-1}$. As $s \rightarrow 0, \beta_{s}$ uniformly converges to $\gamma$; since $\gamma$ is extended through $e$, this means that $\alpha\left(\gamma, \beta_{s}\right) \rightarrow 0$. But then the representative angle $\alpha_{k}\left(e ; \gamma(-s), \gamma(s)^{-1}\right)$, which is smaller than $\alpha\left(\gamma, \beta_{s}\right)$, also tends to 0 . 
Then by definition, $\alpha\left(\gamma^{-1},-\gamma\right)=0$, and so the two geodesics coincide. Now let $s$ be small enough that $\zeta_{s}=\left.\gamma\right|_{[-s, s]}$ is the unique minimal curve between its endpoints. Then the isometry $L_{\gamma(s)}$ takes $\zeta_{s}$ to a unit minimal curve of length $2 s$ starting at $e$ and passing through $\gamma(s)$; therefore $\gamma(s) \zeta_{s}$ coincides with the segment of $\gamma$ from $e$ to $\gamma(2 s)$. Since unit geodesics are uniquely determined by any segment (with interior), for any small enough $s, \gamma$ is invariant under $L_{\gamma(s)}$, and $\gamma(s) \gamma(t)=\gamma(t+s)$. Let $S>0$ be the supremum of all $s$ such that $\gamma(s) \gamma(t)=\gamma(t+s)$ for all $t$, and suppose $S<\infty$. Then $\gamma(3 S / 2)=\gamma(3 S / 4) \gamma(3 s / 4)$, and for any $t$, $\gamma(3 S / 2) \gamma(t)=\gamma(3 S / 4) \gamma(3 S / 4) \gamma(t)=\gamma(3 S / 4) \gamma(t+3 S / 4)=\gamma(t+3 S / 2)$, a contradiction.

Remark 4.1. From now on, if $\gamma$ is a geodesic through $e$, we will denote by $-\gamma$ the geodesic defined by $-\gamma(t)=\gamma(-t)$. For example, it is always true that $\alpha(\gamma,-\beta)=$ $\pi-\alpha(\gamma, \beta)$.

Corollary 4.3. A group with bi-invariant inner metric of curvature bounded below is geodesically complete.

Proof. We need to show that an arbitrary minimal curve $\gamma$ starting at $e$ can be extended as a geodesic through $e$. For any $\gamma(t)$ in the interior of $\gamma, \beta(s)=\gamma(t)^{-1} \gamma(s)$ is a minimal curve through $e$; hence $\beta(s)$ extends to a 1-parameter subgroup. The left invariance of the metric implies that $\beta$ restricted to a small interval about 0 is a minimal curve, call it $\sigma(s)$. But then $\psi(s)=\gamma(t) \psi(s)$ is the desired extension of $\gamma$

Proposition 4.4. If $G$ has a bi-invariant inner metric of curvature bounded below, then $G$ has curvature $\geq 0$.

Proof. Let $\gamma$ and $\beta$ be unit minimal curves starting at $e$ of length $L, M$, respectively. Then for any $s, t, d(\gamma(2 s), \beta(2 t))=d\left(\gamma(s)^{2}, \beta(t)^{2}\right) \leq d\left(\gamma(s)^{2}, \gamma(s) \beta(t)\right)+$ $d\left(\gamma(s) \beta(t), \beta(t)^{2}\right)=2(\gamma(s), \beta(t))$. In other words, $G$ has curvature $\geq 0$ in a "weak" (cf. [24]) sense. Suppose $\alpha(\gamma, \beta)=A$, fix $\epsilon>0$ and choose $n$ large enough that $\left|d\left(\gamma\left(L / 2^{n}\right), \beta\left(M / 2^{n}\right)\right) 2^{n}-\left(L^{2}+M^{2}-2 L M \cos A\right)\right|<\epsilon$. Then from what we proved above, $\left|d(\gamma(L), \beta(M))-\left(L^{2}+M^{2}-2 L M \cos A\right)\right|<\epsilon$. Letting $\epsilon$ go to 0 verifies Alexandrov's comparison condition, and proves the proposition.

We say that a space $X$ with inner metric has positive cut radius if every point $p \in X$ has a neighborhood in which every point is joined to $p$ by a unique minimal curve. It is well-known that in a Lie group with bi-invariant Riemannian metric, geodesics through $e$ and 1-parameter subgroups coincide (cf. [12]). We have the following extension of this result:

Proposition 4.5. If $G$ has a bi-invariant inner metric of curvature $\geq 0$ and positive cut radius then geodesics through e and non-trivial 1-parameter subgroups coincide.

Proof. Let $\gamma$ be a 1-parameter subgroup. Suppose that $c_{t}:[0, t] \rightarrow G$ is minimal from $e$ to $\gamma(t)$. By Proposition $4.2 c$ extends to a 1-parameter subgroup which we call by the same name. Now $c_{t / 2}(t)=\left(c_{t / 2}(t / 2)\right)^{2}=\gamma(t / 2)^{2}=\gamma(t)=c_{t}(t)$. In other words, if $c_{t / 2}$ and $c_{t}$ are distinct then the cut radius of $c_{t / 2}$ is less than $d(e, \gamma(t))$, or twice its length. Since the cut radius of $G$ is positive, for small enough $t$, all the curves $c_{t / n}$ must coincide with a single geodesic $\gamma^{\prime}$. But then $\gamma$ and $\gamma^{\prime}$ 
initially coincide on a dense set of points in their domain of definition, and, being both continuous, must coincide everywhere.

We remark that, under the assumptions of 4.5, $G$ has a neighborhood $U$ of $e$ in which each point $x$ lies on a unique segment of a 1-parameter subgroup in $U$ from $e$ to $x$. That is, $U$ is (uniquely) "ruled" by 1-parameter subgroups. It is a classical (cf. [15]) that a locally compact group having such a neighborhood must be a Lie group.

Let $G$ be a group with invariant metric, $H$ a normal subgroup which is finitely compact (Definition 2.2) with respect to the induced metric. If we identify $H$ with the group of isometries of $G$ consisting of the left translates of elements of $H$, then orbits of this action are precisely the cosets of the quotient group $G / H$.

Proposition 4.6. If $G$ is locally compact and has bi-invariant inner metric of curvature $\geq 0$ then $G$ is weakly convex.

Proof. Since $G$ has an inner metric, $G$ is arcwise connected, and hence connected; this implies that $G / G_{0}$ is (trivially) compact. By the Gleason-Iwasaswa-Yamabe theory of locally compact groups, $G$ is a projective limit of Lie groups. If $G$ is finite dimensional, $G$ is a Lie group with bi-invariant Riemannian metric, and we are finished. Suppose $G$ is infinite dimensioinal. Since $G$ is first countable, that means we can find a countable sequence of properly nested (cf. [9]) normal subgroups $G_{1} \supset G_{2} \supset \cdots$ whose diameters tend to zero, such that $K_{i}=G / G_{i}$ is a Lie group. By Lemma 2.1 the tangent space of $K_{i}$ lifts isomorphically to a subspace $H_{i}$ of $T_{e}$, and $c_{e}$ has a positive lower bound on $S\left(H_{i}\right)=S_{e} \cap H_{i}$ (in fact $c_{e}$ is no smaller than the cut radius of $K_{i}$ on $S\left(H_{i}\right)$. Since $G_{i+1} \subset G_{i}$, Proposition 2.4 implies $T_{i+1}^{\perp} \subset T_{i}^{\perp}$,

and therefore $H_{i}$ is properly contained in $H_{i+1}$. Let $H_{e}=\bigcup_{i=1}^{\infty} H_{i}$. Then $H_{e}$ satisfies the requirements of Definition 1.2 except possibly condition 2). Now let $\gamma \in \bar{S}_{e}$ be arbitrary. Assume first that $c_{e}(\gamma)=T>0$. Fix $\epsilon>0$. For each $i$, there exists $\gamma_{i} \in H_{i}$ from $e$ to the orbit $G_{i} \gamma(T-\epsilon)$. Since $\operatorname{diam}\left\{\left(G_{i} \gamma(T-\epsilon)\right)\right\} \rightarrow 0$ and $G$ is geodesically complete, $\alpha\left(\gamma_{i}, \gamma\right) \rightarrow 0$. Furthermore, $\liminf \left\{c_{e}\left(\gamma_{i}\right)\right\} \geq T-\epsilon$. By letting $\epsilon \rightarrow 0$ and choosing a suitable diagonal subsequence we can choose the $\gamma_{i}$ such that $\lim \inf \left\{c_{e}\left(\gamma_{i}\right)\right\} \geq T$. That $\lim c_{e}\left(\gamma_{i}\right)=T$ now follows from the upper semicontinuity of the cut radius. In particular we have shown that $H_{e}$ is dense in $T_{e}$ and therefore in $\bar{T}_{e}$. Therefore, even if $c_{e}(\gamma)=0$, there exist $\gamma_{i} \rightarrow \gamma$, and $c_{e}\left(\gamma_{i}\right) \rightarrow 0$ again by the upper semicontinuity of $c_{e}$.

\section{Existence of Invariant Weakly Convex Metrics}

Proof of Theorem 1.3. We first claim the following: For any $\epsilon>0$ there exists a $\delta>0$ such that for any $s \leq t$ with $0<s<\delta / 4$ and $t<3 s, \frac{d_{s}+d_{t}-d_{t+s}}{d_{s}}<\epsilon$, where $d_{r}$ denotes $d(e, \gamma(r))$. Let $\gamma$ be unit parameterized, $0<\delta<1 / 2$ be small enough that if $P$ is any partition of $[0,8]$ of norm $\|P\|<\delta$, then the corresponding sum $\Sigma(P)$ satisfies $8-\Sigma(P)<\kappa:=\frac{\epsilon}{1+\epsilon}$. Then for any $a<8$, if $P^{\prime}$ is a partition of $[0, a]$ with $\left\|P^{\prime}\right\|<\delta, a-\Sigma\left(P^{\prime}\right)<\kappa$. Suppose that $\frac{1}{n} \leq s<\frac{1}{n-1}(n \geq 2)$. Then $\frac{2}{n} \leq s+t \leq$ $\frac{4}{n-1}$, and so $n s$ and $n(s+t)$ are both $\leq 8$. Since $\gamma$ is a 1-parameter subgroup and the metric is invariant, for any real numbers $r_{1}<r_{2}, d\left(\gamma\left(r_{1}\right), \gamma\left(r_{2}\right)\right)=d\left(e, \gamma\left(r_{2}-r_{1}\right)\right)$. By using the partition of $[0, n s]$ whose points are integral multiples of $s$, we see that since $s<\delta, n s-n d_{s}<\kappa$, which implies $d_{s}>s-\frac{\kappa}{n} \geq \frac{1-\kappa}{n}$. Similarly, we obtain 
$s+t-d_{s+t}<\frac{\kappa}{n}$. Now $\frac{d_{s}+d_{t}-d_{t+s}}{d_{s}}=\frac{d_{s}-s+d_{t}-t+s+t-d_{t+s}}{d_{s}} \leq \frac{s+t-d_{t+s}}{d_{s}}<\frac{\kappa}{1-\kappa}=\epsilon$, proving the first claim.

We next claim that for any $\epsilon>0$ there exists a $\delta>0$ such that for any $s \leq t$ with $0<s<\delta$ and $t<2 s$, if $\gamma_{r}$ denotes a minimal curve from $e$ to $\gamma(r)$ then $\alpha\left(\gamma_{s}, \gamma_{t}\right)<\epsilon$. In fact, the above claim shows that for small enough $s, \alpha\left(\gamma_{-s}, \gamma_{s}\right)$ and $\alpha\left(\gamma_{-s}, \gamma_{s+t}\right)$ are both arbitrarily close to $\pi$. But then $\alpha\left(\gamma_{s}, \gamma_{s+t}\right)$ must be arbitrarily close to 0 . In particular, we have shown that a sequence of minimal curves $\gamma_{i}$ from $e$ to $\gamma\left(2^{-i}\right)$ is Cauchy (in angle); hence it converges to some direction we will also call $\gamma$.

To complete the proof, consider positive $t_{i} \rightarrow 0$ and let $\beta_{i}$ be minimal curves from $e$ to $\gamma\left(t_{i}\right)$. We need to show that $\beta_{i} \rightarrow \gamma$. But for each $i$, there is some $j$ such that $2^{-j} \leq t_{i}<2^{-j+1}$, and by the second claim, $\alpha\left(\beta_{i}, \gamma_{j+1}\right)$ is arbitrarily small for large $j$. We remark here that we have used the existence of certain minimal curves to prove the proposition. This is not essential if we use the results of [21]. In the case of present interest, the local compactness provides the needed minimal curves.

In the above proof we found $t_{i} \rightarrow 0$ such that $\lim _{i \rightarrow \infty} \sigma\left(e ; \gamma\left(t_{i}\right), \gamma\left(-t_{i}\right)\right)=0$; this implies that the corresponding representative angles must tend to $\pi$ (see the discussion preceding Proposition 2.4):

Corollary 5.1. Let $G$ be a group with left invariant inner metric of curvature $\geq k$ and $\gamma$ be a non-trivial rectifiable 1-parameter subgroup of $G$. Then there exist positive $t_{i} \rightarrow 0$, with $\lim _{i \rightarrow \infty} \alpha_{k}\left(e ; \gamma\left(t_{i}\right), \gamma\left(-t_{i}\right)\right)=\pi$.

Note that the product of invariant or bi-invariant metrics is invariant or biinvariant, respectively.

Proposition 5.2. If $G_{i}$ has a bi-invariant inner metric of curvature $\geq 0$ and positive cut radius, then a curve $\beta$ in $G=\prod_{i=1}^{\infty} G_{i}$ is a 1-parameter subgroup in $G$ if and only if $\beta=\sum_{i=1}^{\infty} k_{i} \gamma_{i}$, where each $\gamma_{i}$ is a unit geodesic $\left(\sum_{i=1}^{\infty} k_{i}^{2}\right.$ may or may not be finite).

Proof. Since every geodesic is a 1-parameter subgroup (Lemma 4.2), any curve of the form $\sum_{i=1}^{\infty} k_{i} \gamma_{i}$ is a 1-parameter subgroup. Conversely, every 1-parameter subgroup $\beta$ in $G$ is of the form $\left(\beta_{1}, \beta_{2}, \ldots\right)$, where each $\beta_{i}$ is a 1-parameter subgroup in $G_{i}$. By Lemma 4.5 each non-trivial $\beta_{i}$ is also a geodesic; so $\beta_{i}=k_{i} \gamma_{i}$ for unit $\gamma_{i}$, and $\beta=\sum_{i=1}^{\infty} k_{i} \gamma_{i}$.

Corollary 5.3. Let $G_{i}$ be groups with bi-invariant inner metrics of curvature $\geq 0$ and positive cut radius. Then there exist non-rectifiable 1-parameter subgroups in $G:=\prod_{i=1}^{\infty} G_{i}$

More generally it follows from the proof of Theorem 1.1 that non-rectifiable 1parameter subgroups exist in any locally compact infinite dimensional group, with the weakly convex metrics we construct. 
Corollary 5.4. If $G_{i}$ has a bi-invariant inner metric of curvature $\geq 0$ and positive cut radius, then in $G=\prod_{i=1}^{\infty} G_{i}$ every non-trivial rectifiable 1-parameter subgroup is tangent to a direction, and every direction is tangent to a unique unit 1-parameter subgroup.

Under the conditions of Corollary 5.4 it makes sense to write $\alpha\left(\beta_{1}, \beta_{2}\right)$ for two rectifiable 1-parameter subgroups; we let $\alpha\left(\beta_{1}, \beta_{2}\right)$ be the angle between the corresponding directions.

Lemma 5.5. Suppose $G_{i}$ has a bi-invariant inner metric of curvature $\geq 0$ and positive cut radius and let $G=\prod_{i=1}^{\infty} G_{i}$. If $\beta, \beta_{j}$ are unit 1 -parameter subgroups such $\alpha\left(\beta, \beta_{j}\right) \rightarrow 0$ then $\beta_{j}$ converges uniformly to $\beta$ on any closed interval.

Proof. Fix a closed interval $[0, a]$. Let $\beta=\sum_{i=1}^{\infty} k_{i} \gamma_{i}$ and $\beta_{j}=\sum_{i=1}^{\infty} k_{j i} \gamma_{j i}$, fix $\epsilon>0$, and choose $M$ such that for any $x, y \in G, d(x, y)^{2}-\sum_{i=1}^{M} d\left(x_{i}, y_{i}\right)^{2}<\epsilon / 2$. By Lemma 3.3, if $\beta^{M}=\sum_{i=1}^{M} k_{i} \gamma_{i}$ and $\beta_{j}^{M}=\sum_{i=1}^{M} k_{j i} \gamma_{j i}$ then $\alpha\left(\beta^{M}, \beta_{j}^{M}\right) \rightarrow 0$. Then for large enough $j$ and $t \in[0, a], \epsilon / 2>d\left(\beta^{M}(t), \beta_{j}^{M}(t)\right)^{2}=\sum_{i=1}^{M} d\left(k_{i} \gamma_{i}(t), k_{j i} \gamma_{j i}(t)\right)^{2}>$ $d\left(\beta(t), \beta_{j}(t)\right)^{2}-\epsilon / 2$. That is, $d\left(\beta(t), \beta_{j}(t)\right)^{2}<\epsilon$.

We finally come to the proof of Theorem 1.1. Because the proof involves some technical details, we first provide a sketch of the argument. The proof is easily reduced to the compact case. For compact groups the strategy is to embed $G$ as a "diagonal" subgroup in the product $P=G / K_{1} \times G / K_{2} \times \cdots$, where each $G / K_{i}$ is a compact Lie group with appropriately chosen bi-invariant Riemannian metric. Here $K_{1} \supset K_{2} \supset \cdots$ is a nested sequence of compact normal subgroups whose intersection is the identity, we embed $G$ as $g \mapsto\left(g K_{1}, g K_{2}, \ldots\right)$, and use the induced inner metric. The main difficulty lies in showing that the induced inner metric is finite. In fact, $G$ can be approximated by the Lie groups $G^{n}=\left(g K_{1}, \ldots, g K_{n}, e, \ldots\right)$, all of which have curvature $\geq 0$. If the inclusion mappings of $G^{n}$ into $P$ were (metric, not Riemannian) isometries, $G^{n}$ would converge to $G$ in the Gromov-Hausdorff sense, and we could appeal to known results ([18]). However, the groups $G^{n}$ are totally geodesic in the factor $G / K_{1} \times \cdots \times G / K_{n}$, but not necessarily convex; the inclusion is locally an isometry on balls of radius $\lambda_{n}$, where $\lambda_{n}$ may tend to 0 as $n$ gets large. In other words, the induced inner metric may always be strictly larger than the induced metric. Nonetheless we can use arguments similar to those in [18] once we know that the diameters of the $G^{n}$ are bounded in the induced inner metric. Control of the metric is accomplished by noting that the arcwise connectedness allows us to assume that the fibers of the maps $G / K_{i} \rightarrow G / K_{i-1}$ are connected. We can then take these maps to be Riemannian submersions, having fibers of as small diameter as we choose. Thus for every minimal curve $\gamma$ joing $e$ and $p$ in $G / K_{i-1}$ there is lift $\widetilde{\gamma}$ in $G / K_{i}$ of $\gamma$ joining $e$ to any point in the fiber over $p$, and $L(\widetilde{\gamma})$ is not much longer than $L(\gamma)$. We then scale the metrics to make the diameters of $G / K_{i}$ square summable, and in this way we gain control of the metrics of the $G^{n}$. 
It is useful to see how this procedure fails with a group that is not arcwise connected. The solenoid, $\Sigma$, is obtained as the inverse limit of the sequence $S^{1} \leftarrow$ $S^{1} \leftarrow \cdots$, where each connecting map is multiplication by two. The fiber in each case is two points. The only possible Riemannian submersions are local isometries, which forces the diameter of $S^{1}$, and that of the fiber, to double in each step.

Proof of Theorem 1.1. We first assume that $G$ is compact and show that $G$ has a bi-invariant metric of non-negative curvature with the desired properties. Let $H_{i}$ be a sequence of compact nested normal subgroups of $G$ such that $G / H_{i}$ is a Lie group and $\bigcap H_{i}=e$. Let $K_{i}$ be the identity component of $H_{i} ; K_{i}$ is a normal subgroup of $G$. Then the natural mapping $G / K_{i} \rightarrow G / H_{i}$ has totally disconnected kernel, so $G / K_{i}$ has at most the same (finite) dimension as $G / K_{i}$ (cf. [13]). Since $G$ is arcwise connected, $G / K_{i}$ is also arcwise connected, and is therefore a Lie group (cf. [23]). Note that the kernel of the natural map $\pi_{(i+1) i}$ : $G / K_{i+1} \rightarrow G / K_{i}$ is connected, being the image of the connected group $K_{i}$, under the projection $\pi_{i+1}: G \rightarrow G / K_{i+1}$. Now the universal cover of $G / K_{i}$ is of the form $S_{1} \times \cdots \times S_{k_{i}} \times R_{1} \times \cdots \times R_{n_{i}}$, where each $S_{j}$ is a compact simple Lie group and $R_{j}$ is a copy of the reals (cf. [12]). We choose a bi-invariant Riemannian metric $g_{1}$ on $G / K_{1}$ of diameter smaller than $1 / 2$, which lifts to a Riemannian metric $\widetilde{g}_{1}$ on the universal cover of $G / K_{1}$. We write the universal cover of $G / K_{2}$ as $M_{1} \times M_{2}$ where

$$
\begin{gathered}
M_{1}=\left(S_{1} \times \cdots \times S_{k_{1}} \times R_{1} \times \cdots \times R_{n_{1}}\right), \\
M_{2}=\left(S_{k_{1}+1} \times \cdots \times S_{k_{2}} \times R_{n_{1}+1} \times \cdots \times R_{n_{2}}\right) .
\end{gathered}
$$

On $M_{1}$ have the metric $\widetilde{g}_{1}$. On $M_{2}$ we choose a bi-invariant metric $\widetilde{h}_{2}$, and provide $G / K_{2}$ with the metric $g_{2}$ induced by $\widetilde{g}_{2}=\widetilde{g}_{1} \times k \widetilde{h}_{2}$, where $k$ is a constant to be determined as follows. Since the fibers of the submersion $\pi_{21}$ are the translates of the compact, connected, totally geodesic kernel of this quotient map, by choosing $k$ small enough we can ensure that the fibers have diameter $<1$ with respect to the induced inner (equivalently Riemannian) metric. Note that the projection $\pi_{21}$ is a Riemannian submersion for any choice of $k$; as pointed out in Section 2, the distance from the Riemannian metric on $\left(G / K_{1}, g_{1}\right)$ is identical to the quotient metric from Definition 2.1. Moreover, for each geodesic $\gamma$ starting at $a \in\left(G / K_{1}, g_{1}\right)$ and $b \in \pi_{21}^{-1}(a)$ there is a unique isometric ("horizontal") lift of $\gamma$ to a geodesic $\Gamma$ starting at $b$. We now consider the product $P_{2}=\left(G / K_{1}, g_{1}\right) \times\left(G / K_{2}, \frac{1}{2} g_{2}\right)$, with the product metric, and the "diagonal" subspace $G_{2}=\left\{\left(\pi_{21}(z), z\right)\right\}=\left\{\pi_{1}(x), \pi_{2}(x)\right.$ : $x \in G\} \subset P_{2}$ (which is a closed Lie subgroup of the product), with the induced inner metric (equivalently, the induced Riemannian metric). Suppose that $\left(\pi_{1}(x), \pi_{2}(x)\right)$ and $\left(\pi_{1}(y), \pi_{2}(y)\right.$ are in $G_{2}$ and let $\gamma_{1}$ be minimal from $\pi_{1}(x)$ to $\pi_{1}(y)$ in $G / K_{1}$ of length $L=d\left(\pi_{1}(x), \pi_{1}(y)\right)$. The unique horizontal lift $\Gamma_{1}$ of $\gamma_{1}$ at $\pi_{2}(x)$ is minimal of length $L / 2$ (since we have scaled the metric) and ends at a point $z$ in the fiber above $\pi_{1}(y)$. By our choice of $k$, we can find a geodesic $\beta_{1}$ in the fiber above $\pi_{1}(y)$ joining $z$ to $\pi_{2}(y)$, with $L(\beta)<1 / 2$. The concatenated curve $\gamma_{2}=\Gamma_{1} * \beta_{1}$ joins $\pi_{2}(x)$ and $\pi_{2}(y)$ with length $\frac{L+1}{2}$, and $\pi_{21}\left(\gamma_{2}\right)$ coincides with $\gamma_{1}$ up to reparameterization. That is, $\left(\pi_{21}\left(\gamma_{2}\right), \gamma_{2}\right)$ lies in $G_{2}$, joins $\left(\pi_{1}(x), \pi_{2}(x)\right)$ and $\left(\pi_{1}(y), \pi_{2}(y)\right)$, and has length $<L+\frac{L}{2}+\frac{1}{2}=\frac{3 L+1}{2}$. (Here we use the fact that the length of a curve in a product is at most the sum of the lengths of its projections.) The diameter of $G_{2}$ with this metric is $<5 / 4$. 
We continue this procedure. Choose a Riemannian metric $g_{3}$ on $G / K_{3}$ so that the mapping $\pi_{32}$ is a Riemannian submersion having fibers of diameter smaller than 1. Let $G_{3}=\left\{\left(\pi_{1}(x), \pi_{2}(x), \pi_{3}(x)\right): x \in G\right\} \subset P_{3}=\left(G / K_{1}, g_{1}\right) \times\left(G / K_{2}, \frac{1}{2} g_{2}\right) \times$ $\left(G / K_{3}, \frac{1}{4} g_{3}\right)$. If $a=\left(\pi_{1}(x), \pi_{2}(x), \pi_{3}(x)\right), b=\left(\pi_{1}(y), \pi_{2}(y), \pi_{3}(y)\right) \in G_{3}$ then we can lift the curve $\gamma_{2}$ constructed above to a piecewise geodesic $\sigma$ in $G / K_{3}$ of length $<$ $\frac{L+1}{4}$, and concatenate with a geodesic lying entirely in the fiber above $\pi_{2}(y)$, which has length $<\frac{1}{4}$. Thus we can join $a$ and $b$ in $G_{3}$ by a piecewise geodesic of length $<L+\left(\frac{L}{2}+\frac{1}{2}\right)+\left(\frac{L}{4}+\frac{1}{4}+\frac{1}{4}\right)$. With similar definitions for $G_{i}$, we can join any two points $\left(\pi_{1}(x), \ldots, \pi_{i}(x)\right),\left(\pi_{1}(y), \ldots, \pi_{i}(y)\right) \in G_{i}$ by a curve $\gamma_{i}$ in $G_{i}$ whose projections $\gamma_{i}^{j}$ onto $G / K_{j}$ are piecewise geodesics of length $<\left(2^{-j+1}\right) L+(j-1) 2^{-j+1}, 1 \leq j \leq i$. Then $L\left(\gamma_{i}\right)<2 L+2$, and the diameter of $G_{i}$ is smaller than 3. Note also that the diameter of $\left(G / K_{n}, g_{n}\right)$ is smaller than $\frac{2 n-1}{2}$, so each factor $\left(G / K_{n}, 2^{-n+1} g_{n}\right)$ has diameter smaller than $(2 n-1) 2^{-n}$.

Let $P=\left(G / K_{1}, g_{1}\right) \times\left(G / K_{2}, \frac{1}{2} g_{2}\right) \times \cdots$ with the product metric $d$. We define homomorphisms $\phi_{n}, \phi: G \rightarrow P$ by $\phi_{n}(g)=\left(\pi_{1}(g), \ldots, \pi_{n}(g), e, e, \ldots\right)$ and $\phi(g)=$ $\left(\pi_{1}(g), \ldots\right)$. Note that $\phi$ is a monomorphism, so we can algebraically identify $G$ with its image in $P$. We will show that $\phi(G)$ has the desired metric. First, $\phi_{n}(G)$ lies in the convex subgroup $G / K_{1} \times \cdots \times G / K_{n}$, and (with the induced inner metric) is isometric to $G_{n}$. That is, $\phi_{n}(G)$ has curvature $\geq 0$ and diameter $<3$. Furthermore, $d\left(\phi_{n}(g), \phi(g)\right)<\operatorname{diam}\left\{\left(G / K_{n+1}, 2^{-n+1} g_{n+1}\right) \times \cdots\right\} \rightarrow 0$, so the Hausdorf distance from $\phi_{n}(G)$ to $\phi(G)$ tends to 0 . We will show that the induced (bi-invariant) inner metric on $\phi(G)$ has curvature $\geq 0$. Let $d_{n}$ denote the induced inner metric in $\phi_{n}(G)$ and $d^{\prime}$ denote the induced inner metric in $\phi(G)$. Note that the lengths of curves in $P$ is the same, whether measured in $d, d_{n}$, or $d^{\prime}$. For any $x=\phi(a), y=\phi(b) \in \phi(G)$, choose $x_{n}, y_{n} \in \phi_{n}(G)$ so that $d\left(x_{n}, x\right) \rightarrow 0$ and $d\left(y_{n}, y\right) \rightarrow 0$. Let $\gamma_{n}$ be minimal (in $\left(\phi_{n}(G), d_{n}\right)$ ) from $x_{n}$ to $y_{n}$ defined on $[0,1]$. Since the diameter of $\phi_{n}(G)$ is $<3$, so is the length of $\gamma_{n}$. We can now apply Ascoli's Theorem to find a uniform limit $\gamma$ in $\phi(G)$ of a subsequence $\left\{\gamma_{n_{j}}\right\}$ of $\left\{\gamma_{n}\right\}$. Now let $\Pi$ be a partition of $\gamma$. Then we can approximate $\Pi$ arbitrarily closely by partitions of $\gamma_{n_{j}}$ ( $j$ large); i.e., the sum the distances given by $\Pi$ is smaller than $3-\epsilon, \epsilon>0$ arbitrarily small. In other words, the length $L(\gamma) \leq \liminf L\left(\gamma_{n_{j}}\right)<3$; we see that the induced inner metric on $\phi(G)$ is finite, and has diameter $<3$. We also have that $d^{\prime}(x, y) \leq \liminf d_{n}\left(x_{n}, y_{n}\right)$ whenever $x_{n} \rightarrow x$ and $y_{n} \rightarrow y$. On the other hand, if $\gamma=\left(\gamma_{1}(t), \gamma_{2}(t), \ldots\right):[0,1] \rightarrow \phi(G)$ is minimal, then the curve $\gamma^{n}=$ $\left(\gamma_{1}(t), \ldots, \gamma_{n}(t), e, e, \ldots\right)$ is a shorter curve in $\phi_{n}(G)$, and $\gamma^{n}(0) \rightarrow \gamma(0)$, and $\gamma^{n}(1) \rightarrow$ $\gamma(1)$. That is, if $x^{n}=\left(\pi_{1}(a), \ldots, \pi_{n}(a), e, e, \ldots\right)$ and $y^{n}=\left(\pi_{1}(b), \ldots, \pi_{n}(b), e, e, \ldots\right)$, then

$$
d^{\prime}(x, y)=\lim d_{n}\left(x^{n}, y^{n}\right)
$$

It is now an immediate consequence of the definition that, since each $\left(\phi_{n}(G), d_{n}\right)$ has curvature $\geq 0$, so does $\left(\phi(G), d^{\prime}\right)$.

We next need to verify that the topology on $\phi(G)$ from the induced inner metric $d^{\prime}$, and the induced (subspace) topology on $\phi(G)$ coincide. We use the same notation as in the preceding paragraph. Since $\phi_{n}(G)$ is totally geodesic, there exists a constant $\lambda_{n}>0$ such that if $w, z \in \phi_{n}(G)$ and $d(w, z) \leq \lambda_{n}$, then $d_{n}(w, z)=$ $d(w, z)$. Given $\epsilon>0$, note that if $n$ is large enough, then the sum of the diameters of the factors $\left(G / K_{i}, 2^{-i} g_{i}\right)$, with $i \geq n$ is $<\epsilon / 3$; for any $p=\left(p_{1}, \ldots\right), q=\left(q_{1}, \ldots\right) \in P$, $0 \leq d(p, q)-d_{n}\left(p^{n}, q^{n}\right)<\epsilon / 3$. We can choose $n$ also large enough that $2^{-n+1}<\epsilon / 3$. 
Suppose, as above, that $x=\phi(a), y=\phi(b)$. If $d(x, y)<\epsilon / 3$ is small enough, then $\lambda_{n} \geq d\left(x^{n}, y^{n}\right)$, so $d_{n}\left(x^{n}, y^{n}\right)=d\left(x^{n}, y^{n}\right)$. We have that $0 \leq d(x, y)-$ $d_{n}\left(x^{n}, y^{n}\right) \leq \epsilon / 3$. Also, $0 \leq d_{n+1}\left(x^{n+1}, y^{n+1}\right)-d_{n}\left(x^{n}, y^{n}\right) \leq d_{n}\left(x^{n}, y^{n}\right) / 2+2^{-n}$. In fact, we can proceed as before: let $\gamma$ be minimal in $\phi_{n}(G)$ from $x^{n}$ to $y^{n}$ (in this case, by the way we chose $x$ and $y, \gamma$ is also minimal in $P$ ). We then lift $\gamma$ horizontally and concatenate with a geodesic in the fiber above $y$, which has length $<2^{-n}$. Continuing in this way, we see that, since $d^{\prime}(x, y)=\lim d_{i}\left(x^{i}, y^{i}\right)$, $d^{\prime}(x, y)-d_{n}\left(x^{n}, y^{n}\right) \leq 2 \epsilon / 3$, and $0 \leq d^{\prime}(x, y)-d(x, y)<\epsilon$.

We can now identify $G$ with the topological group $\phi(G)$, which is given the the induced inner metric defined above, of curvature $\geq 0$. By Propositions 4.2, 4.4, 4.6, and Theorem 1.3 we need only verify that every direction $\gamma \in \bar{S}_{e} G$ is tangent to a unique 1-parameter subgroup in $G$. From the last paragraph we know that for $x, y \in G, d(x, y) \leq d^{\prime}(x, y)$, and that the metrics are equivalent in $G$. We denote by $\alpha$ the angle metric in $\bar{S}_{e} P$ and by $\alpha^{\prime}$ the angle metric in $\bar{S}_{e} G$. Choose geodesic directions $\gamma_{i} \in S_{e} G$ converging to $\gamma$. Then each $\gamma_{i}$ corresponds to a geodesic in $G$ (also denoted $\gamma_{i}$ ), which is a rectifiable 1-parameter subgroup. Note that since $\gamma_{i}$ is rectifiable in $\left(G, d^{\prime}\right)$, it is rectifiable in $(P, d)$ (of the same length). We write $\gamma_{i}=$ $\sum k_{i k} \gamma_{i k}$ as in Proposition 5.2. We claim that for any $i, j$, if $\gamma_{i}^{n}=\sum_{k=1}^{n} k_{i k} \gamma_{i k}, \gamma_{j}^{n}=$ $\sum_{k=1}^{n} k_{j k} \gamma_{j k}$, then $\alpha^{\prime}\left(\gamma_{i}, \gamma_{j}\right)=\lim _{n \rightarrow \infty} \alpha\left(\gamma_{i}^{n}, \gamma_{j}^{n}\right)=\alpha\left(\gamma_{i}, \gamma_{j}\right)$. (Here the last equality is Proposiion 3.10.) The first equality can be proved using the geodesic completeness in essentially the same way as the proof of Proposition 9 in [18]: From the equation (5.1) and the way angles are defined we know that $\alpha^{\prime}\left(\gamma_{i}, \gamma_{j}\right) \leq \lim _{n \rightarrow \infty} \alpha\left(\gamma_{i}^{n}, \gamma_{j}^{n}\right)$. Applying the same argument to complementary directions, we obtain the opposite inequality.

By choosing a diagonal subsequence of the geodesics $\left\{\gamma_{i}^{n}\right\}$ we can construct a sequence $\left\{\alpha_{i}=\sum h_{i k} \alpha_{i k}\right\}$, Cauchy in $S_{e} P$, such that $\alpha\left(\alpha_{i}, \alpha_{j}\right)-\alpha^{\prime}\left(\gamma_{i}, \gamma_{j}\right)<2^{-i}$, $i \leq j$ and $\alpha\left(\alpha_{i}, \gamma_{i}\right)<2^{-i}$. By Proposition 3.11 we have that $\lim _{k \rightarrow \infty} h_{i k}=\lim _{k \rightarrow \infty} k_{i k}:=$ $m_{i}$ and $\lim _{i \rightarrow \infty} \alpha_{i}=\lim _{i \rightarrow \infty} \gamma_{i}:=\beta_{i}$ (here we may have to change our representations in case $\left.\beta_{i}=0\right)$. By Lemma 5.5, the unit 1-parameter subgroup $\beta=\sum m_{i} \beta_{i}$ lies in the (closed) subgroup $G$. We need to show that $\gamma$ is tangent to $\beta$. Let $\xi_{i}$ be minimal in $G$ from $e$ to $\beta(1 / i)$. Theorem 1.3 implies that the sequence $\left\{\xi_{i}\right\}$ is Caucy in $S_{e} G$. Since $\xi_{i}$ is also a rectifiable 1-parameter subgroup, an immediate consequence of Proposition 3.11 is that $\left\{\xi_{i}\right\}$ and $\left\{\gamma_{i}\right\}$ have the same limit in $\bar{S}_{e} G$, namely $\gamma$. Uniqueness follows from the fact that our construction is independent of the choice of the sequence $\left\{\gamma_{i}\right\}$. This completes the proof of Theorem 1.1 in the compact case. We note here that our argument implies that $\bar{S}_{e} G$ can be considered as a Hilbert subspace of $\bar{S}_{e} P$ in a natural way.

If $G$ is not compact, the Gleason-Iwasawa-Yamabe theory (as distilled in [9]) implies that there exists a neighborhood $U$ of $e$ in $G$ such that $U=H \times V$, where $H$ is a compact group and $V$ is a local Lie group. Put an invariant Riemannian metric on $V$, and restrict to a compact, convex neighborhood $W$ of $V$. If we now put a metric on $H$ of the type constructed above, then we have metrized $H \times W$ as a compact neighborhood of the identity of $G$. We metrize all of $G$ in the following way. For any curve $c$ in $G$, we measure the length of $c$ as follows: Divide $c$ into segments short enough that if the endpoint of a segment is left translated to $e$, then the image of the entire segment lies in $H \times W$. We can measure the length of the segment there, and define the length of $c$ to be the sum of the lengths of the segments. It is easy to see that the length of $c$ is well defined. Letting $d(x, y)$ be the 
infimum of lengths of curves measured in this way, it follows that $d$ is an invariant intrinsic metric locally isometric to the metric on $H \times G$. Since the properties we need to verify are all local, the proof is complete.

The author would like to thank V. N. Berestovskii and C. Stallmann for useful discussions about this paper, and the referee for his patience with my corrections and revisions.

\section{REFERENCES}

[1] Alexandrov, A.D., Die innere Geometrie der konvexen Flächen, Akad. Verl., Berlin, 1955. MR 17:74

[2] Alexandrov, A.D., Berestovskii, V.N., and Nikolaev, I.G., Generalized Riemannian spaces, Russian Math Surveys 41 (1986) 1-54. MR 88e:53103

[3] Berestovskii, V. N., Spaces with bounded curvature and distance geometry, Siberian Math. J. 27 (1986) 8-19. MR 89k:53063

[4] Berestovskii, V. N., Homogeneous spaces with intrinsic metric, Soviet Math. Dokl. 27 (1989) 60-63.

[5] Berestovskii, V. N., and Plaut, C., Homogeneous spaces of curvature bounded below, preprint.

[6] Cheeger, J., and Ebin, D., Comparison theorems in Riemannian geometry, North-Holland Math Library 9 (1975), Amsterdam. MR 56:16538

[7] Burago, Yu., Gromov, M., and Perelman, G., A.D. Alexandrov spaces with curvature bounded below, Russian Math Surveys 47 (1992) 1-58. MR 93m:53035

[8] Cohn-Vossen, S., Existenz kürzester Wege, Dokl. Math. SSSR 8 (1935) 339-342.

[9] Gluskov, V.N., The structure of locally compact groups and Hilbert's Fifth Problem, AMS Translations 15 (1960) 55-94. MR 22:5690

[10] Gromov, M., Lafontaine, J., and Pansu, P., Structures métrique pours les variétés riemanniannes, Textes Math., vol. 1 CEDIC/Fernand Nathan, 1981. MR 85e:53051

[11] Grove, K., Metric differential geometry, Differential Geometry, Lecture Notes in Math., vol. 1263, Springer (1987) 171-227. MR 88i:53075

[12] Helgason, S., Differential Geometry, Lie Groups, and Symmetric Spaces, Academic Press, New York, 1978. MR 80k:53081

[13] Hurewicz, W. and Wallman, H., Dimension Theory, Princeton U. Press, Princeton, 1941. MR 3:312b

[14] Lashof, R., Lie algebras of locally compact groups, Pac. J. Math. 7 (1957) 1145-1162. MR 19:1064a

[15] Montgomery, D. and Zippin, L., Topological transformation groups, Interscience, New York, 1955. MR 17:383b

[16] O'Neill, B., The fundamental equations of a sumersion, Michigan Math J. 13 (1966), 459-469. MR 34:751

[17] Plaut, C., Almost Riemannian spaces, J. Diff. Geom. 34 (1991) 515-537. MR 92h:53047

[18] Plaut, C., Metric curvature, convergence, and topological finiteness, Duke Math. J. 66 (1992) 43-57. MR 93e:53051

[19] Plaut, C., Metric pinching of locally symmetric spaces, Duke Math. J. 73 (1994) 155-162. MR 95c:53055

[20] Plaut, C., Correction to "Metric pinching of locally symmetric spaces", Duke Math. J., 75 (1994) 527-528. CMP 94:17

[21] Plaut, C., Spaces of Wald curvature bounded below, J. Geom. Analysis, to appear.

[22] Rickert, N. W., Arcs in Locally Compact Groups, Math. Annalen 172 (1967), 222-228. MR 35: 4331

[23] Rickert, N., Some Properties of Locally Compact Groups, J. Austral. Math. Soc. 7 (1967) 433-454. MR 36:2735

[24] Rinow, W., Die Innere Geometrie der Metrischen Räume, Springer, Berlin, 1961. MR 23:A1290

[25] van Kampen, E. R., The structure of a compact connected group, Amer. J. Math. 57 (1935) 301-308.

Department of Mathematics, University of Tennessee, Knoxville, Tennessee 37996 1300

E-mail address: plaut@novell.math.utk.edu 